\title{
Contribución al estudio del consumo específico de energía, en la molienda de los materiales de la industria del cemento, según el ensayo de F. C. Bond
}

\author{
FERNANDO MORENO ARUS Y JULIO PEREZ ALONSO \\ Laboratorio Central de Ensayos de la Companila General \\ de Asfaltos y Portland Asland
}

\section{INTRODUCCION}

Desde un principio la industria del cemento conoció la necesidad de un método con el que se pudiera determinar. En primer lugar, la molturabilidad de los materiales empleados (materias primas, crudos, clínkeres, correctores del cemento, etc.), mediante un ensayo normalizado que permitiera una independización del sistema de molienda empleado, lográndose así una verdadera estandarización del ensayo, del que se obtendría una medida cuantitativa de la respuesta del material al esfuerzo de la molienda. Y en segundo lugar, a partir de la molturabilidad, el poder determinar en función de la granulometría de la alimentación y del producto terminado el consumo energético previsto en una instalación industrial.

En este sentido, desde que Taggart afirmó que un hombre experimentado con un yunque y un martillo puede decir más sobre la molturabilidad de un material, que un hombre inexperto, que haga un control de molturabilidad, han mejorado mucho las cosas, y los pronósticos exactos del valor de la molienda son una realidad.

De todos los métodos aparecidos, el ensayo de Bond es el usado por mayor número de fabricantes de equipos y es el que presenta más ventajas frente a los otros. El proceso de trituración en el molino Bond concuerda con el proceso de molienda en el molino industrial en su mayor parte, ya que en ambos molinos se producen los mismos tipos de esfuerzo, la cantidad necesaria para el ensayo es relativamente pequeña y la carga de bolas es constante en contraposición con otros métodos.

El método elegido por nosotros ha sido el "Bond Ball Mill" que consiste en un ensayo a escala de laboratorio, con un molino de bolas normalizado, en seco y simulando un circuito cerrado con una carga circulante del $250 \%$, mediante una separación periódica de los finos por un tamiz. Y se ha aplicado sistemáticamente para determinar la dureza de 
los diferentes materiales que usualmente se someten a molienda con lo que, actualmente, se empieza a disponer de un número de resultados que permite poner en consideración el método aplicado.

\section{METODO "BOND BALL MILL"}

\subsection{Fundamento teórico}

Está basado en los tres principios de la trituración y molienda y la tercera teoría de F. C. Bond que exponemos a continuación.

\section{Primer Principio}

La energía suministrada en la trituración y molienda o energía absorbida por el material, para obtener la reducción del tamaño de la alimentación, es igual a la diferencia del contenido energético del producto final menos la energía del material alimentado.

\section{SEgundo Principio}

El trabajo necesario para la trituración y molienda es proporcional a la longitud de la grieta producida, lo que para partículas de forma parecida equivale a la diferencia $1 / \sqrt{\mathrm{P}}-1 / \sqrt{a}$, donde $\mathrm{P}$ es el diámetro de las partículas del producto y $a$ es el diámetro de las partículas de la alimentación.

\section{Tercer Principio}

Establece que la facilidad de agrietamiento en una partícula determina la resistencia a la rotura, pero no su índice de trabajo.

Este viene dado por la relación entre la granulometría de las partículas antes y después del agrietamiento.

Las variaciones del índice de trabajo dependen de la mayor o menor cantidad de grietas producidas, las cuales originan distintos tamaños de partículas a partir de los tamaños de origen.

\section{Tercera Teoria de la Molienda}

Basándose en los tres principios de la molienda; F. C. Bond propone la siguiente ecuación, mediante la cual se determina el trabajo teórico (T. t.) necesario para la trituración y molienda de un material desde una granulometría a otra.

$$
\text { T. t. }=\frac{10 \mathrm{IT}}{\sqrt{\mathrm{P}}}-\frac{10 \mathrm{IT}}{\sqrt{\mathrm{A}}}(\mathrm{I})
$$

o introduciendo la relación de trituración $\mathrm{R}=\mathrm{A} / \mathrm{P}$ :

$$
\text { T. t. }=\left(1-\frac{1}{\mathrm{R}^{0,5}}\right)\left(\frac{100^{0,5}}{\mathrm{P}}\right)
$$


Para los cálculos prácticos el tamaño de partícula que corresponde al tamiz que permite el paso del $80 \%$ del material es el que se fija como el diámetro, bien sea del material de alimentación, o el producto molido; por lo tanto:

$\mathrm{A}=$ Luz del tamiz en micras que retiene el $20 \%$ (o deja pasar el $80 \%$ ) del producto de la molienda.

$\mathrm{P}=$ Luz del tamiz en micras que retiene el $20 \%$ de la alimentación al molino.

IT = Indice de trabajo: es el parámetro de trituración y molienda, que expresa la resistencia del material al machaqueo y molienda, y nos dice los $\mathrm{kW} \cdot$ hora/t necesarios para reducir un material de alimentación desde un tamiz técnicamente infinito, hasta que pase un $80 \%$ por el tamiz de 100 micras.

Según la fórmula empírica desarrollada por F. C. Bond: [1].

Donde:

$$
\mathrm{IT}=\frac{16}{\mathrm{G}^{0,82}} \sqrt{\frac{\gamma}{100}}
$$

IT $=$ Indice de trabajo.

$\mathrm{G}=$ Molturabilidad $\mathrm{g} /$ revolución.

$\gamma=$ Tamaño de la malla en micras del tamiz separador.

Teniendo en cuenta que $\mathrm{G}$ es función lineal de $\gamma$, del tipo $\mathrm{G}=a \gamma+b$, siendo tanto más acusado este hecho, cuanto mayor es la heterogeneidad del material a ensayar. Vemos que realmente el índice de trabajo no es un parámetro constante.

Proponiendo Holmes [2] para evitar el considerar este carácter variable, el aceptar el exponente de trituración como no conocido, con lo cual la ecuación de Bond se transforma en:

$$
\text { T. t. }=\mathrm{IT}\left(1-\frac{1}{\mathrm{R}^{\mathrm{r}}}\right)\left(\frac{100}{\mathrm{P}}\right)^{\mathrm{r}}
$$

\subsection{Parte experimental - Método de ensayo}

El método se realiza con un molino de bolas de $30,48 \times 30,48 \mathrm{~cm}$, con velocidad de 70 rpm y una carga de $20.125 \mathrm{~g}$ de bolas de $3,810-2,540$ y $1,905 \mathrm{~cm}$, repartida en 27 bolas de $3,810 \mathrm{~cm}, 57 \mathrm{de} 2,540 \mathrm{~cm}$, y $201 \mathrm{de} 1,905 \mathrm{~cm}$. Durante el ensayo se mantiene la carga de material con un volumen constante de $700 \mathrm{~cm}^{3}$.

El material a ensayar, $10 \mathrm{~kg}$, secado en la estufa, se reduce a tamaño inferior a $3,2 \mathrm{~mm}$ con una machacadora de mandibulas mediante un primer machaqueo, del que se separa lo mayor de $3,2 \mathrm{~mm}$, que se vuelve a machacar hasta que sea inferior, mezclando bien el material ya terminado.

El ensayo se continúa, determinando, en la tamizadora vibratoria, el porcentaje del material menor de 90 micras para su uso en posteriores cálculos.

El tamiz usado para la separación de finos, en los diferentes períodos del ensayo, fue el de 4.900 mallas $/ \mathrm{cm}^{2}$, o sea 90 micras (realmente se puede establecer el índice de trabajo para cualquier tamiz, aunque F. C. Bond lo define para el de 100 micras). 
La carga del molino $700 \mathrm{~cm}^{3}$ de material a ensayar se midió en una probeta de plástico graduada de $1.000 \mathrm{~cm}^{3}$, llenándola en cuatro etapas; en la primera se colocan $250 \mathrm{~cm}^{3} \mathrm{y}$ se dan 10 golpes soltando la probeta de plástico desde $3 \mathrm{~cm}$ sobre una superficie dura; en la segunda etapa se rellena la probeta hasta $500 \mathrm{~cm}^{3}$ y se dan otros 10 golpes; en la tercera se rellena hasta $700 \mathrm{~cm}^{3}$ y 10 golpes y, finalmente, en la cuarta etapa, se enrasa hasta $700 \mathrm{~cm}^{3}$.

La carga del molino se efectúa siempre, colocando primero las bolas y después el material a ensayar, evitando así efectos molturadores previos.

Para establecer una carga circulante del $250 \%$, por cada gramo de material producto molido que sale del separador (que en este caso es el tamiz de 90 micras), tienen que volver 2,5 g de material, con lo que la alimentación, es 3,5 g para cada gramo producido; por lo tanto, para establecer esta carga circulante, tenemos que producir unos g que son los de la carga del molino equivalentes a $700 \mathrm{~cm}^{3}$ divididos por $3,5 \mathrm{~g}$, y a este valor, se le denomina producción prevista para un período (P.P.P.).

El primer período del ensayo se inicia dando 100 revoluciones al molino cargado con los $700 \mathrm{~cm}^{3}$ de material a ensayar, al final de las cuales, se quita todo el material del molino, separando, mediante un tamiz de $10 \mathrm{~mm}$ la carga de bolas de la carga de material, de la que mediante la tamizadora vibratoria, se separarán los finos inferiores a 90 micras, determinándose el peso del residuo sobre el tamiz y, restado del peso de la carga (de $700 \mathrm{~cm}^{3}$ de volumen), se obtiene el peso del material producto molido.

El peso del material inferior a $90 \mu$ después de la molienda, llamado producto molido, menos lo menor de 90 micras introducido en la carga, da los gramos netos de material producido en este primer período, con lo que dividiendo estos gramos producidos por las 100 revoluciones efectuadas, obtenemos los $\mathrm{g} / \mathrm{rev}$. para el primer período.

En el segundo período, se carga el molino con los superiores a 90 micras que quedaron del primer período, más una nueva alimentación del material preparado inferior a 3,2 $\mathrm{mm}$, equivalente en peso a los finos inferiores a 90 micras eliminados al final del primer período.

Para calcular las revoluciones a realizar, es necesario restar al P.P.P. los gramos inferiores introducidos en la alimentación, y dividir los g así obtenidos por los g/rev. obtenidos en el primer período.

Calculadas las revoluciones para el segundo período, se procede a efectuarlas, y separar al final de las mismas los gramos inferiores a 90 micras, de los que deducidos los presentes, antes de la molienda, obtendremos los realmente producidos, que a su vez divididos por las revoluciones realizadas dan los g/rev. para el segundo período.

Así se continúa hasta que se obtienen tres períodos consecutivos en los que los g/rev. producidos son sensiblemente iguales (lo usual es que se estabilice con valores comprendidos en un intervalo de $\pm 0,2$ ), de los que se efectúa el promedio para hallar los gramos por revolución o molturabilidad.

A partir de la molturabilidad se determina el índice de trabajo mediante la fórmula (II) para $\gamma=90$ micras.

Aplicando el método tal cual hemos descrito, hemos logrado una reproductividad desde $\pm 0,01 \mathrm{~g} / \mathrm{rev}$., sobre muestras duras como el clínker, a $\pm 0,04$ en muestras blandas como la diatomita (exceptuando las arcillas, las cuales son prácticamente imposible de ensayar por falta de reproductividad). 


\subsection{Discusión del método utilizado}

El propósito del ensayo de molturabilidad es obtener una comparación de la resistencia de molienda de materiales diferentes, que pueden ser rápidamente reducidos a la potencia de energía requerida. No es necesario, para esta comparación, que el producto de la molienda tenga una distribución de tamaños similar a los productos terminados industriales, sin embargo, se desea que la distribución de tamaños de los productos molidos (menores de 90 micras) sean similares dentro de las diferentes clases de materiales, crudos entre sí, clínkeres, etc.

Esto generalmente se consigue, pues la separación en el tamiz de 90 micras es eficaz y se obtiene una carga circulante muy ajustada al $250 \%$, con una carga de bolas y velocidad del molino también constante. Aunque, esta similitud puede ser comprobada cribando el producto final del ensayo por el tamiz de 325 mallas, haciendo granulometrías o medidas de superficie específica.

Así, cuando existe apreciable diferencia entre los materiales ensayados, es suficiente, para que los resultados de ambos sean comparativos, que las curvas granulométricas de la alimentación y el producto terminado mantengan una relación.

A pesar de todo lo anteriormente expuesto F. C. Bond [3] propone una fórmula análoga a la (II), con un factor de corrección en el que intervienen los tamices que dejan pasar el $80 \%$ de la alimentación $(a)$ y del producto del último período $(\mathrm{P})$, quedando la siguiente expresión:

$$
\mathrm{IT}=\frac{44,5}{\gamma^{0,23} \cdot \mathrm{G}^{0,82} \cdot-\frac{10}{\sqrt{\mathrm{P}}}-\frac{10}{\sqrt{a}}}
$$

Nosotros, en la serie de ensayos realizados, no hemos tenido en cuenta esta corrección (que implica, en la práctica, efectuar la granulometría por tamices, para la alimentación y la granulometría mediante un fluorómetro del producto inferior a 90 micras), aceptando que dentro de cada uno de los grupos establecidos, debido a la homogeneidad de la granulometría entre las materias que lo componen, obtendríamos una concordancia relativamente buena, al aplicar las fórmulas (II) y (III).

Finalmente, para los casos en que el material a ensayar no es homogéneo, en cuanto a la dureza de sus componentes, -mezclas de clínker y yeso por ejemplo- se nos ha presentado un fenómeno, ya citado en la bibliografía, de clasificación, enriqueciéndose en yesos los finos inferiores a 90 micras presentes en los primeros períodos, como se comprueba al determinar el contenido en $\mathrm{SO}_{3}$ de la alimentación, y en las fracciones superior e inferior a 90 micras de los dos primeros períodos.

M U E S T R A

$\% \mathrm{SO}_{3}$

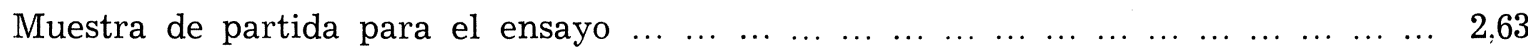

Fracción inferior a 4.900 mallas (90 micras después del primer período) $\ldots \ldots \ldots \ldots$. . . $\quad 5,17$

Fracción inferior a 4.900 mallas después del $2 .^{\circ}$ período $\begin{array}{llllllllllllll}\ldots & \ldots & \ldots & \ldots & \ldots & \ldots & \ldots & \ldots & \ldots & 3,73\end{array}$

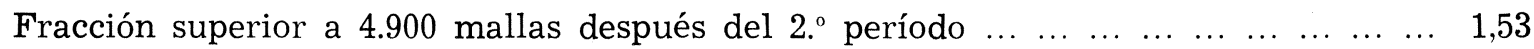


Este efecto de clasificación creemos nos ha repercutido en un falseamiento de las molturabilidades de estas mezclas de clínker y yeso para las que hemos obtenido valores de g/rev. inferiores a los del clínker sólo, contrariamente a lo que era de esperar.

Idéntico fenómeno de clasificación se ha producido al determinar la molturabilidad de mezclas de clínker con materiales blandos en la fabricación de cementos especiales, en este ensayo también el componente blando de alto residuo insoluble en clorhídrico, aparece en mayor proporción en los finos de los primeros períodos, según se puede deducir de los siguientes datos:

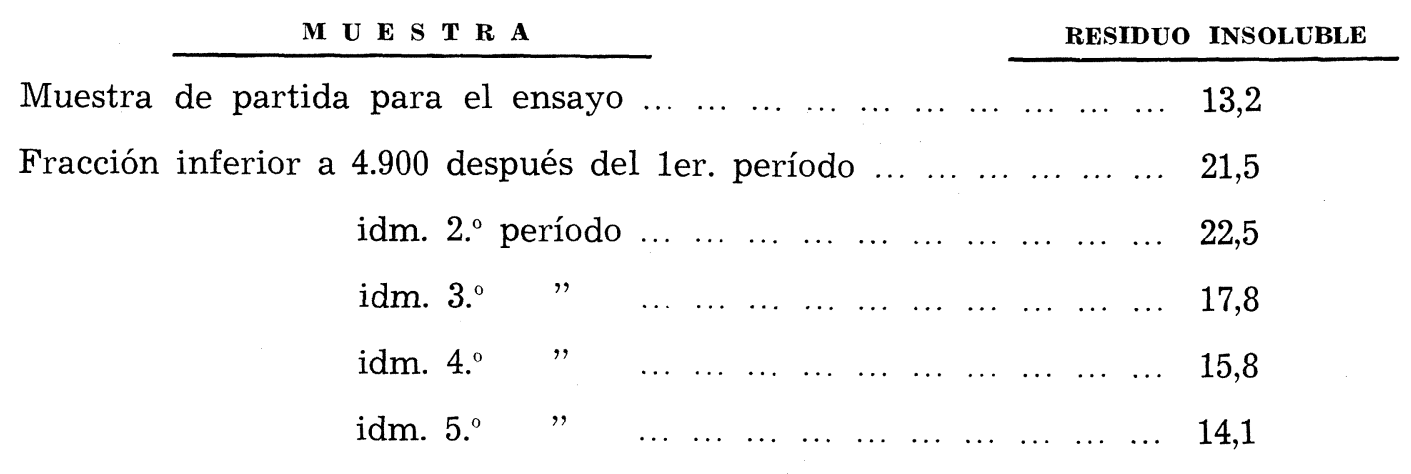

\section{ENSAYOS REALIZADOS Y DISCUSION DE RESULTADOS}

\subsection{Materiales ensayados}

Con las características de los materiales ensayados clasificados en materias primas, crudos y clínker, y los correspondientes índices de trabajo, se confeccionaron las tablas I, II y III respectivamente.

\subsubsection{Materias primas}

Los resultados obtenidos con los 28 materiales ensayados vienen en la tabla I y en los gráficos Ia y Ib, en las que destacan como materias duras: el granito, las limburgitas, escorias, los basaltos de Massanet, la tierra de Salone, los lapillis de Olot y las arenas, siguiéndoles en este orden las calizas, entre las cuales las más duras son la Jurásica Dogger y Cretácica Inferior, las más blandas Mioceno Pontiense y los finos de calizas Silúricas.

Se ha buscado la relación de la molturabilidad con el grado de saturación potencial, el módulo de silicatos, el de fundentes y también con el contenido en cuarzo (determinado por Difracción de rayos X); hallando la correlación lineal simple entre el índice de trabajo y cada una de las variables citadas (tabla I bis). Resultando tan solo significativa la correspondiente al cuarzo, en el sentido de que al aumentar su contenido aumenta el consumo de energía por tonelada (gráfico Ic y Id).

\subsubsection{Crudo}

Con un total de 37 crudos ensayados, ver tabla II y gráficos IIa y IIb; de los que se desprende que el orden de menor a mayor molturabilidad es el siguiente; Fca. n. ${ }^{\circ}$, $2,1,3$ y 5,4 y 6 . 
T A B L A I

\begin{tabular}{|c|c|c|c|c|c|c|c|}
\hline \multirow{2}{*}{ Material } & \multicolumn{7}{|c|}{ 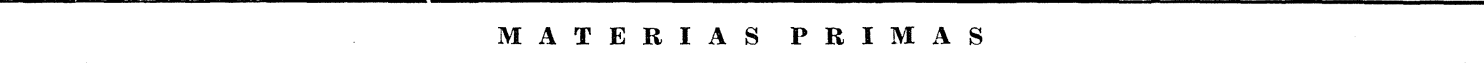 } \\
\hline & $\mathrm{g} / \mathbf{r e v}$ & kW $\cdot$ hora $/ \mathbf{t}$ & Cuarzo & G.S.P. & M.s. & M.F. & Selección \\
\hline Arena de Bundseistein & 0,84 & 17,6 & 72 & 0,4 & 19,76 & 1,36 & $\mathrm{a}$ \\
\hline " Triásica & 0,87 & 17,1 & 55 & 0,9 & 13,19 & 9,60 & a \\
\hline Pizarra Silúrica & 1,69 & 9,9 & 25 & 0,6 & 1,89 & 2,29 & \\
\hline Caliza alta magnesiana Silúrica & 1,33 & 12,0 & & & & & \\
\hline Caliza media $" \quad "$ & 1,29 & 12,3 & 4 & 117 & 1,10 & 0,66 & $\mathrm{a}$ \\
\hline Finos caliza Silúrica & 1,63 & 10,1 & 5 & 38 & 14,82 & 1,20 & $\mathrm{a}$ \\
\hline Caliza Cretácica Inferior & 1,16 & 13,4 & - & 576,6 & 1,13 & 1,60 & \\
\hline " Jurásica Dogger & 0,92 & 16,3 & 一 & 372,6 & 1,6 & 1,6 & \\
\hline$" \quad$ Eoceno & 1,42 & 11,4 & - & $2.612,-$ & 1,09 & 0,83 & \\
\hline " Mioceno Pontiense & 2,13 & 8,2 & & & & & \\
\hline Marga Eoceno & 1,62 & 10,2 & - & 29 & 3,11 & 2,71 & \\
\hline$" \quad$ vindoboniense & 2,1 & 8,3 & & & & & \\
\hline Lapillis Olot & 0,76 & 18,5 & & & & & \\
\hline Basaltos Terciarios Massanet & 0,75 & 19,2 & & & & & \\
\hline$" \quad$ Silúrico & 1,0 & 15,2 & & & & & \\
\hline Limburgitas de Ciudad Real & 0,72 & 19,9 & & & & & \\
\hline Tierra de Santorin & 1,2 & 13,1 & & & & & \\
\hline$"$ de Segni & 3,25 & 12,6 & & & & & \\
\hline " de Sacrófano & 1,74 & 9,6 & & & & & \\
\hline$" \quad$ de Salone & 0,79 & 18,4 & & & & & \\
\hline Pegmatita de Vivero & 1,05 & 14,6 & & & & & \\
\hline Piedra Pómez & 1,53 & 10,7 & & & & & \\
\hline Diatomita Eocénica & 3,9 & $4,9 \quad(1)$ & & & & & \\
\hline Escoria & 0,73 & 19,6 & - & 33,3 & 3,14 & 57,5 & \\
\hline Puzolana artificial & 1,83 & 9,2 & & & & & \\
\hline Yeso Triásico & 2,43 & 7,3 & & & & & \\
\hline$" \quad$ Mioceno de espejo & 2,09 & 8,3 & & & & & \\
\hline Granito & 0,68 & 20,0 & & & & & \\
\hline
\end{tabular}

(1) Media de los tres ensayos efectuados sobre la misma muestra. 
ARENA BUNDSEISTEIN

” TRIASTICA

PIZARRA SILURICA

CALIZA ALTA MAGNESIANA SILURICA

" MEDIA

FINOS CALIZA SILURICA

CALIZA CRETACICA INFERIOR

" JURASICA DOGgER

" EOCENO

ग MIOCENO PONTIENSE

MARGA EOCENO

" VINDOBONIENSE

LAPILLIS OLOT

BASALTOS TERCIARIOS MASSANET

"SILURICOS

LIMBURGITAS DE CIUDAD REAL

TIERRA DE SANTORIN

"SEGNI

" SACROFANO

" SALONE

PEGMATITA DE VIVERO

PIEDRA POMEZ

DIATOMITA EOCENICA

ESCORIA

PUZOLANA ARTIFICIAL

YESO TRIASICO

" MIOCENO DE ESPEJO GRANITO

(c) Consejo Superior de Investigaciones Científicas Licencia Creative Commons 3.0 España (by-nc)

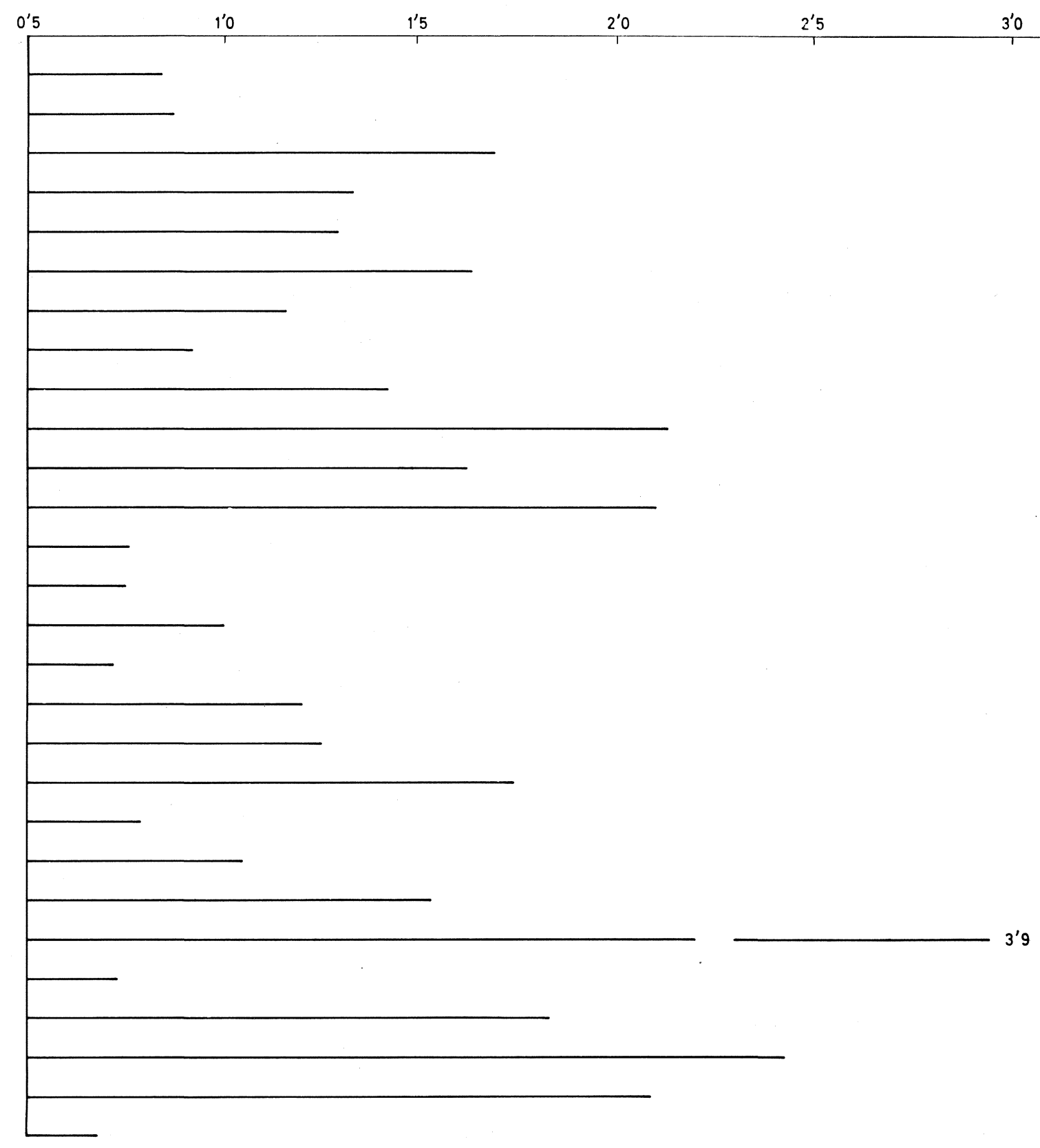

GRAFICO I a 


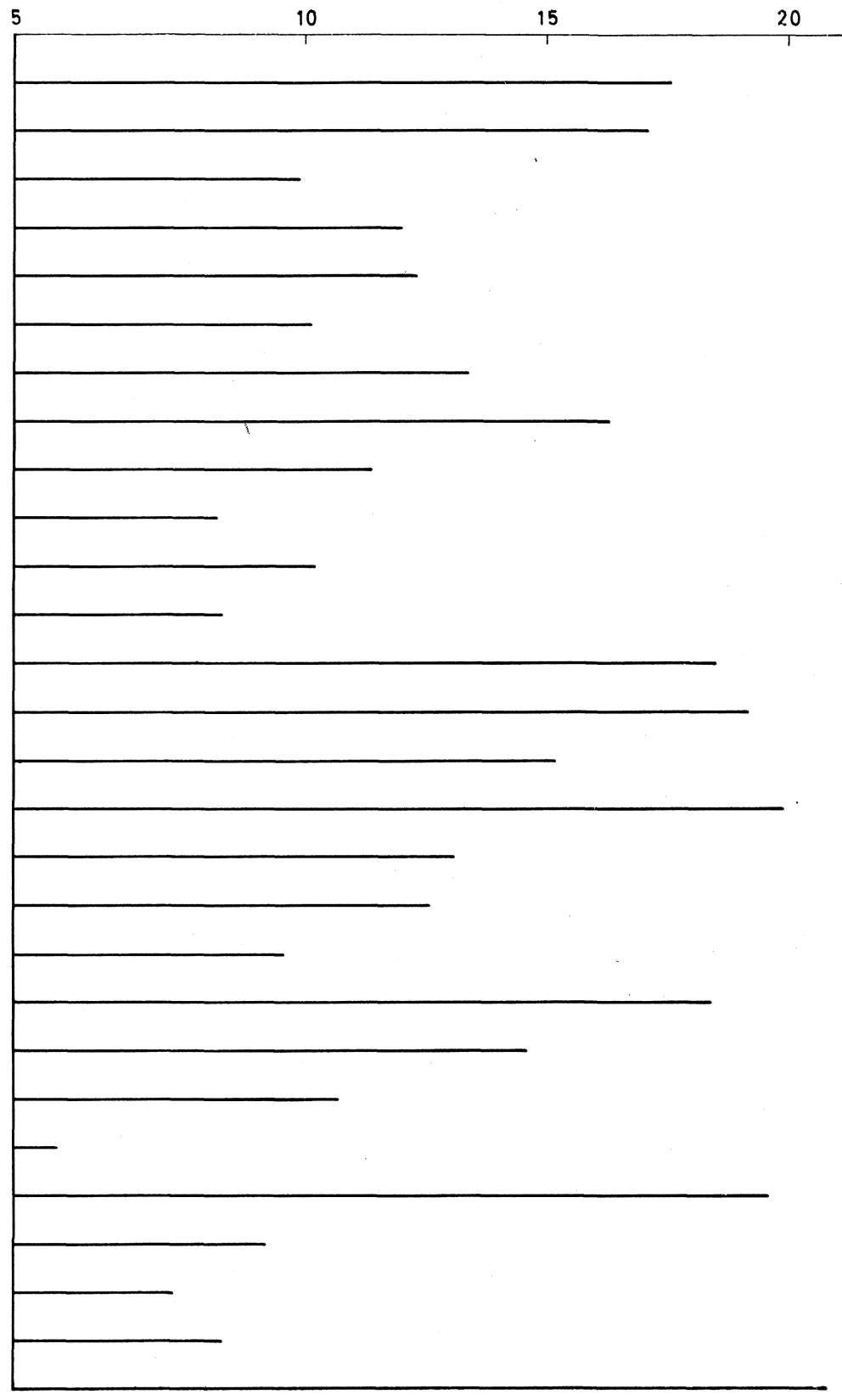

ARENA BUNDSEISTEIN

"TRIASICA

PIZARRA SILURICA

CALIZA ALTA MAGNESIANA SILURICA

"MEDIA " "

FINOS CALIZA SILURICA

CALIZA CRETACICA INFERIOR

" JURASICA DOgger

" EOCENO

" MIOCENO PONTIENSE

MARGA EOCENO

" VINDOBONIENSE

LAPILLIS OLOT

BASALTOS TERCIARIOS MASSANET

$$
\text { ग SILURICO }
$$

LIMBURGITAS DE CIUDAD REAL

TIERRA SANTORIN

" DE SEGNI

" SACROFANO

" SALONe

PEgMATITA DE VIVERO

PIEDRA POMEZ

DIATOMITA EOCENICA

ESCORIA

PUZOLANA ARTIFICIAL

YESO TRIASICO

" MIOCENo de ESPEjo

GRANITO

GRAFICO I b 
T A B L A I bis

\begin{tabular}{|c|c|c|c|c|c|}
\hline & & Cuarzo & G.S.P. & M.s. & M.F. \\
\hline $\begin{array}{l}\text { Con la totalidad de los } \\
\text { valores de la tabla } \mathrm{I}\end{array}$ & $\begin{array}{l}\text { Coef. cor. } \\
\text { Pendiente } \\
\text { Ord. orig. } \\
\text { t. calculado }> \\
\text { t. tabulado }\end{array}$ & $\begin{array}{c}0,87 \\
0,11 \\
9,93 \\
\\
\text { si }\end{array}$ & $\begin{array}{r}0,06 \\
0,00 \\
13,64 \\
\text { no }\end{array}$ & $\begin{array}{r}0,28 \\
0,14 \\
12,92 \\
\\
\text { no }\end{array}$ & $\begin{array}{r}0,61 \\
0,12 \\
12,80 \\
\\
\mathrm{si}\end{array}$ \\
\hline $\begin{array}{l}\text { Selección }(a) \text {. Tomando } \\
\text { solamente los valo res } \\
\text { marcados con } a \text {, en la } \\
\text { tabla I }\end{array}$ & $\begin{array}{l}\text { Coef. cor. } \\
\text { Pendiente } \\
\text { Ord. orig. } \\
\text { t. calculado }> \\
\text { t. tabulado }\end{array}$ & $\begin{array}{l}0,87 \\
0,11 \\
9,93 \\
\\
\text { si }\end{array}$ & $\begin{array}{r}-0,33 \\
-0,02 \\
14,17 \\
\text { no }\end{array}$ & $\begin{array}{r}0,61 \\
0,27 \\
10,61 \\
\text { no }\end{array}$ & $\begin{array}{r}0,51 \\
0,51 \\
11,85 \\
\text { no }\end{array}$ \\
\hline
\end{tabular}

$\mathrm{t}=$ Coeficiente del Test de Student.

Coef. cor. = Coeficiente correlación.

Ord. orig. = Ordenada en origen.

Considerando los valores de la tabla II en su totalidad, no se obtiene ninguna correlación lineal simple significativa, aunque tomando los valores señalados con $(a)$ vemos que existe una correlación muy débil, que resulta ser directa, para el índice de trabajo frente al módulo de fundentes y el módulo de silicatos respectivamente, ver tabla II bis.

T A B L A II bis

\begin{tabular}{|c|c|c|c|c|}
\hline & & G.S.P. & M.s. & M.F. \\
\hline Total & $\begin{array}{l}\text { Coef. cor. } \\
\text { Pendiente } \\
\text { Ord. orig. } \\
\text { t calculado }> \\
\text { t. tabulado }\end{array}$ & $\begin{array}{r}0,02 \\
0,02 \\
10,42 \\
\text { no }\end{array}$ & $\begin{array}{r}-0,27 \\
-3,50 \\
22,85 \\
\text { no }\end{array}$ & $\begin{array}{r}0,25 \\
3,45 \\
6,07 \\
\\
\text { no }\end{array}$ \\
\hline Selección $(a)$ & $\begin{array}{l}\text { Coef. cor. } \\
\text { Pendiente } \\
\text { Ord. orig. } \\
\text { t calculado }> \\
\text { t. tabulado }\end{array}$ & $\begin{array}{r}0,06 \\
0,02 \\
9,21 \\
\\
\text { no }\end{array}$ & $\begin{array}{r}-0,62 \\
-2,23 \\
17,75 \\
\text { si }\end{array}$ & $\begin{array}{r}-0,72 \\
-3,24 \\
16,49 \\
\mathrm{si}\end{array}$ \\
\hline
\end{tabular}

\subsubsection{Clínker}

Los clínkeres ensayados fueron 41, agrupados en la tabla III y gráficos IIIa y IIIb, en los que aparece como clínker más molturable el de la fábrica $n .^{\circ} 7$; del que se ha estudiado su comportamiento con el envejecimiento, resultando un incremento considerable de su índice de trabajo.

Es de notar que en los ensayos sobre mezclas de clínker y yeso dan una molturabilidad con un consumo del orden de $1 \mathrm{~kW}$. hora/t, más que el clínker solo, aspecto ya comentado en el apartado 2.3.

Las restantes adiciones estudiadas son:

a) Limburgitas (Ciudad Real), que por tener una molturabilidad parecida al clínker no alteran el índice de trabajo de la mezcla. 
b) Las puzolanas artificiales y tierras fosilizadas que disminuyen el índice de trabajo de sus mezclas respectivas con el clínker en función de sus molturabilidades.

En cuanto a la influencia de las características del clínker en su molturabilidad.

De todas las variables dependientes consideradas frente al índice de trabajo tomado como variable independiente (tabla III bis y gráficos IIIc, d, e y f) presentan correlación lineal simple significativa:

Variable dependiente

Módulo de silicatos

Fase líquida, calculada según la fórmula PM Lea y TW Parker a $1.400^{\circ} \mathrm{C}$

$\%$ Fase intersticial determinada por recuento planimétrico

$\%$ Belita, determinada por recuento planimétrico

$\frac{\begin{array}{c}\text { Tipo de } \\ \text { correlación }\end{array}}{\text { mediana }}$

mediocre

mediana

mediocre

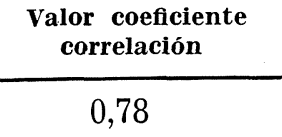

0,58

0,76

0,61

Aunque en el caso de la belita la recta de regresión tiene una pendiente de $-0,09$ lo que realmente nos indica que la influencia de esta variable es nula, para los valores considerados.

Las correlaciones efectuadas con los valores de la selección (a), dan como significativa a las mismas variables más el M. F. obteniendo valores de los coef. de correlación iguales o superiores.

Estos mismos valores de la selección $(a)$ se han considerado también en correlación lineal múltiple, con dos variables dependientes frente a la independiente, del índice de trabajo, con lo que vemos la influencia de la segunda variable dependiente introducida. Ver tabla III bis, de la que extraemos los siguientes valores:

\begin{tabular}{|c|c|c|c|}
\hline $\begin{array}{c}\text { Variable } \\
\text { independiente }\end{array}$ & $\begin{array}{l}\text { primera variable } \\
\text { dependiente }\end{array}$ & $\begin{array}{c}\text { segunda variable } \\
\text { dependiente }\end{array}$ & Coef. corr. \\
\hline \multirow[t]{16}{*}{$\mathrm{kW} \cdot$ hora $/ \mathrm{t}$} & MS & - & 0,78 \\
\hline & & GSP & 0,81 \\
\hline & MF & - & 0,76 \\
\hline & & MS & 0,78 \\
\hline & & GSP & 0,81 \\
\hline & FL & - & 0,75 \\
\hline & & MF & $0,76^{\prime}$ \\
\hline & & MS & 0,78 \\
\hline & & GSP & 0,79 \\
\hline & F. Int. & - & 0,76 \\
\hline & & GSP & 0,79 \\
\hline & & Belita & 0,79 \\
\hline & & Alita & 0,80 \\
\hline & & FL & 0,85 \\
\hline & & MS & 0,87 \\
\hline & 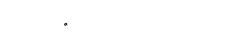 & MF & 0,87 \\
\hline
\end{tabular}




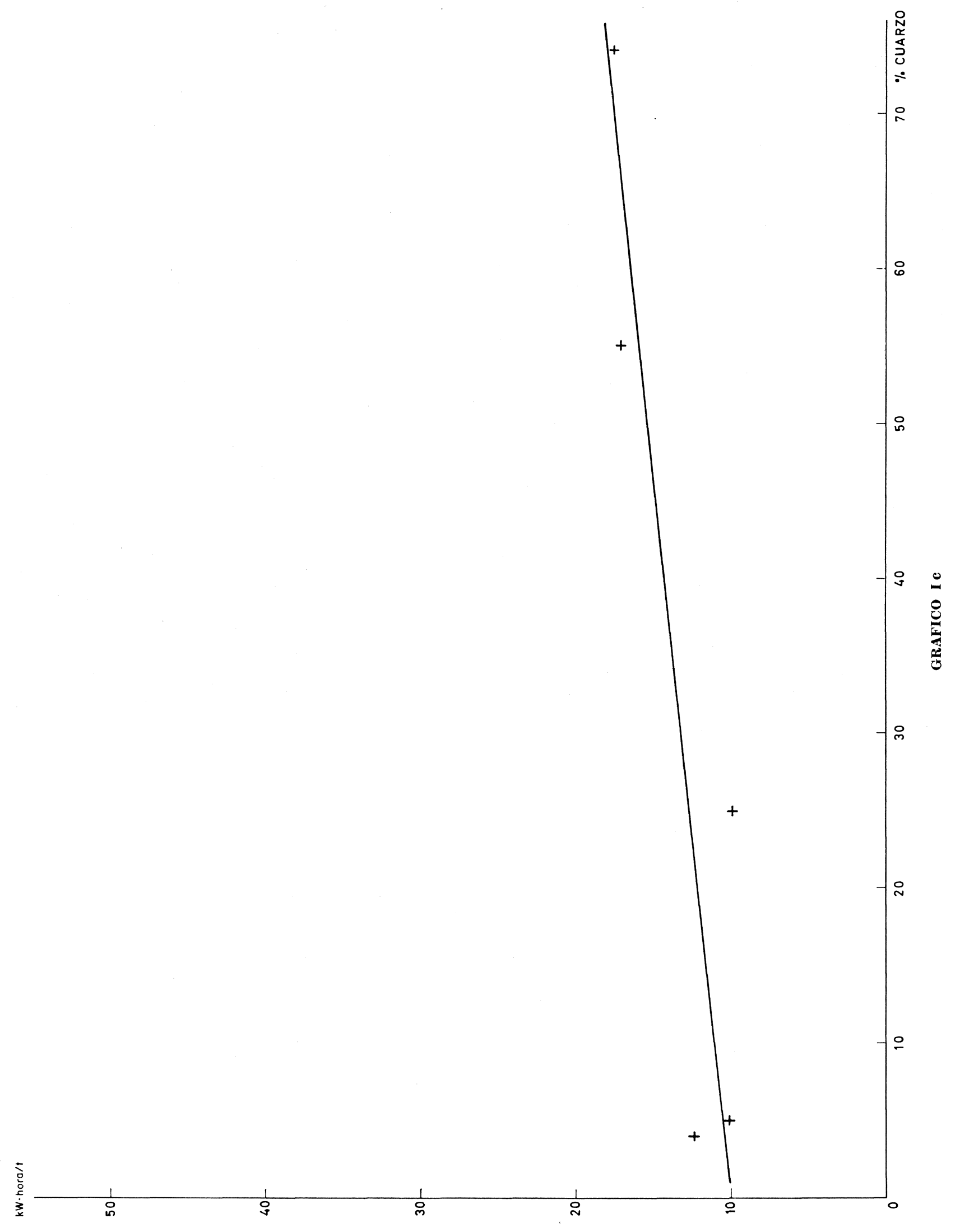




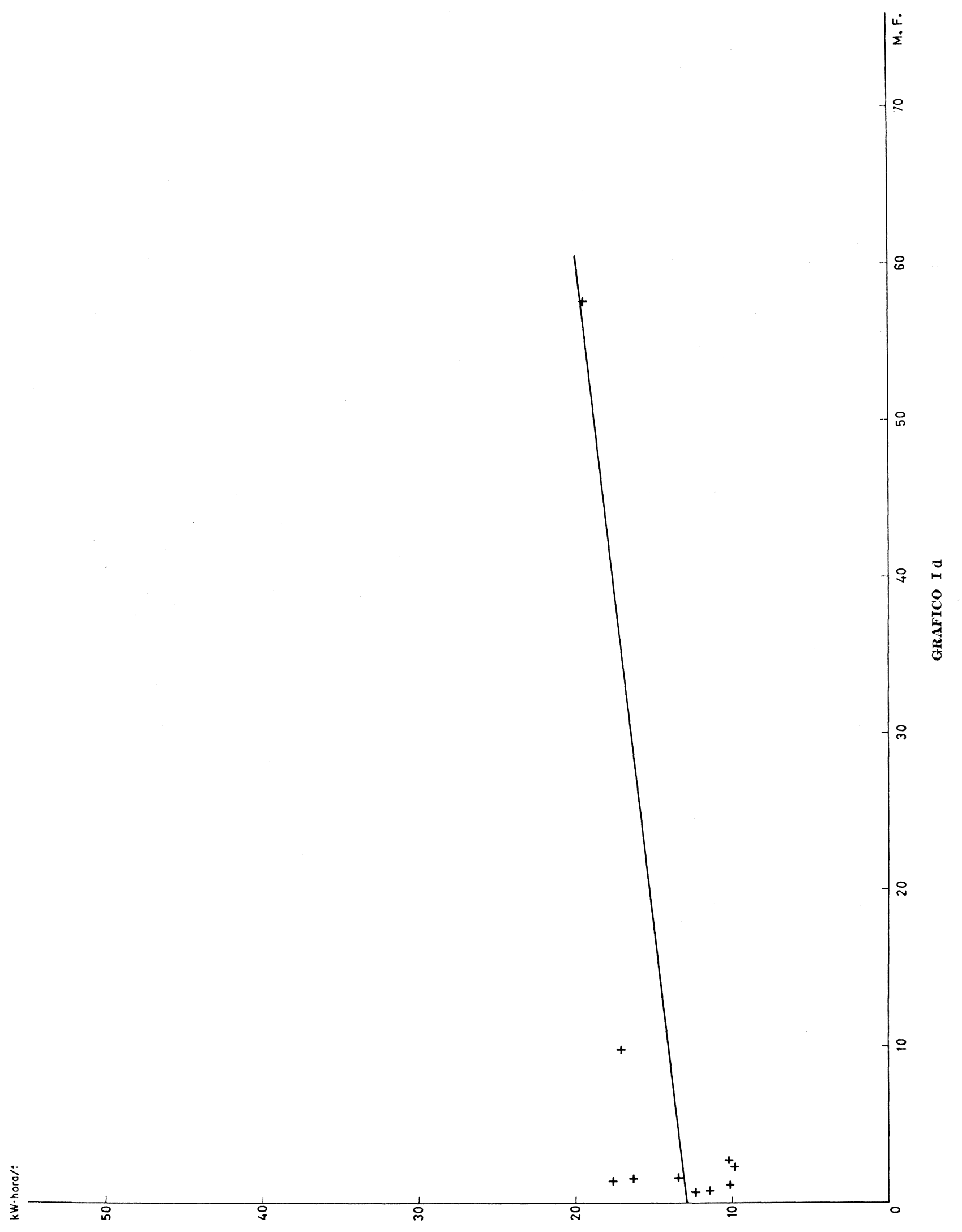

35 
TABLA II

\begin{tabular}{|c|c|c|c|c|c|c|c|c|c|c|c|c|c|}
\hline MATERIAL & & & MPONENTES & & & & $\mathrm{g} / \mathrm{rev}$ & kW . hora/t & \% Cuarzo & G.S.P. & M.s. & M.F. & Selección \\
\hline Fábrica n.o 1 & $\begin{array}{l}\text { Caliza } \\
\text { Eoceno }\end{array}$ & $\begin{array}{l}\text { Marga } \\
\text { Eoceno }\end{array}$ & $\begin{array}{l}\text { Arcilla Vin- } \\
\text { doboniense }\end{array}$ & & & & & & & & & & \\
\hline 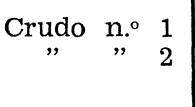 & $\begin{array}{l}54,75 \\
76,35\end{array}$ & $\stackrel{45,25}{-}$ & 23,65 & & & & $\begin{array}{l}1,59 \\
1,61\end{array}$ & $\begin{array}{l}10,4 \\
10,3\end{array}$ & $=$ & $\begin{array}{l}94,1 \\
94,2\end{array}$ & $\begin{array}{l}3,4 \\
2,8\end{array}$ & $\begin{array}{l}2,5 \\
2,7\end{array}$ & \\
\hline Fábrica n.o 2 & $\begin{array}{c}\text { Caliza } \\
\text { cretácica } \\
\text { inferior }\end{array}$ & $\begin{array}{c}\text { Caliza } \\
\text { media } \\
\text { magnesiana } \\
\text { silúrica }\end{array}$ & $\begin{array}{l}\text { Pizarra } \\
\text { silưrica }\end{array}$ & $\begin{array}{c}\text { arena } \\
\text { triásica }\end{array}$ & $\mid \begin{array}{c}\text { arena } \\
\text { Bundseistein }\end{array}$ & & & & & & & & \\
\hline $\begin{array}{ccc}\text { Crudo } & n_{;}^{\circ} & 1 \\
\# & 2\end{array}$ & $\begin{array}{l}67,87 \\
67,45\end{array}$ & $\begin{array}{l}14,0 \\
14,5\end{array}$ & $\begin{array}{r}10,04 \\
8,78\end{array}$ & $\begin{array}{l}7,55 \\
8.45\end{array}$ & $=$ & $\begin{array}{l}0,54 \\
0,82\end{array}$ & $\begin{array}{l}1,20 \\
1,18\end{array}$ & $\begin{array}{l}13,1 \\
13,2\end{array}$ & 7,2 & $\begin{array}{l}94,0 \\
940\end{array}$ & 2,5 & 1,5 & $\mathrm{a}$ \\
\hline $\begin{array}{lll}\prime \prime & \cdots & 3 \\
\end{array}$ & 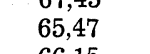 & 17,12 & 6,87 & 9,42 & - & 1,12 & $\begin{array}{l}l, 110 \\
1,17\end{array}$ & $\begin{array}{l}1,2,2 \\
13,3\end{array}$ & 7.,5 & $\begin{array}{l}94,0 \\
94,0\end{array}$ & $\begin{array}{l}2,5 \\
2,5\end{array}$ & $\begin{array}{l}1,8 \\
1,1\end{array}$ & $\begin{array}{l}\mathrm{a} \\
\mathrm{a}\end{array}$ \\
\hline $\begin{array}{lll}\prime \prime & 4 & 4 \\
\end{array}$ & $\begin{array}{l}66,15 \\
65,76\end{array}$ & $\begin{array}{l}16,45 \\
17,03\end{array}$ & $\begin{array}{l}9,44 \\
7,10\end{array}$ & $\begin{array}{l}7,96 \\
9,47\end{array}$ & $\overline{-}$ & $\begin{array}{l}0,34 \\
0,69\end{array}$ & $\begin{array}{l}1,15 \\
1,16\end{array}$ & $\begin{array}{l}13,5 \\
13,4\end{array}$ & $\begin{array}{l}4,7 \\
7,7\end{array}$ & $\begin{array}{l}94,0 \\
94.0\end{array}$ & $\begin{array}{l}2,7 \\
2,7\end{array}$ & $\begin{array}{l}1,5 \\
1,3\end{array}$ & $\mathrm{a}$ \\
\hline 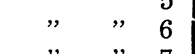 & 65,53 & $\begin{array}{l}17,37 \\
17,37\end{array}$ & $\begin{array}{r}1,108 \\
1,69\end{array}$ & $\begin{array}{l}10,39 \\
10,39\end{array}$ & $\bar{x}$ & 1,03 & $\begin{array}{l}l, 10 \\
1,20\end{array}$ & $\begin{array}{l}13,4 \\
13,1\end{array}$ & , & 94,0 & $\begin{array}{l}2,1 \\
2,7\end{array}$ & $\begin{array}{l}1,1 \\
1,1\end{array}$ & a \\
\hline $\begin{array}{lll}\prime & \cdots & 7 \\
\cdots & 0 & 8\end{array}$ & $\begin{array}{l}66,43 \\
67,12\end{array}$ & 16,10 & 10,94 & 二 & $\begin{array}{l}6,35 \\
698\end{array}$ & $\begin{array}{l}0,18 \\
0,60\end{array}$ & 1,18 & 13,2 & 8,0 & 94,0 & 2,5 & 1,5 & $\mathrm{a}$ \\
\hline $\begin{array}{lll}\prime & n & 0 \\
9\end{array}$ & $\stackrel{67,12}{-}$ & $\stackrel{15,31}{-}$ & $\stackrel{9,99}{-}$ & 二 & & - & $\begin{array}{l}1,24 \\
1,17\end{array}$ & $\begin{array}{l}1,1 \\
13,3\end{array}$ & - & $=$ & 二 & - & \\
\hline Fábrica n.o 3 & $\begin{array}{c}\text { Caliza } \\
\text { mioceno }\end{array}$ & $\begin{array}{c}\text { Marga } \\
\text { pontiense }\end{array}$ & & & & & & & & & & & \\
\hline $\begin{array}{ccc}\text { Crudo } & \text { n.o } & 1 \\
\# & \# & 2\end{array}$ & & & & & & & $\begin{array}{l}1,70 \\
1,77\end{array}$ & $\begin{array}{l}9,8 \\
9,5\end{array}$ & $\begin{array}{l}9,0 \\
7,6\end{array}$ & $\begin{array}{r}79.8 \\
107,2\end{array}$ & $\begin{array}{l}3,3 \\
3, \mathrm{e}\end{array}$ & $\begin{array}{l}1,8 \\
2,1\end{array}$ & \\
\hline Fábrica $n$ n.o 4 & $\begin{array}{c}\text { Caliza } \\
\text { mioceno }\end{array}$ & $\begin{array}{c}\text { Marga } \\
\text { mioceno }\end{array}$ & & $\begin{array}{l}\text { Cenizas } \\
\text { piritas }\end{array}$ & & & & & & & & & \\
\hline Crudo $\mathrm{n}^{\circ} 1$ & 81,34 & 16,76 & 1,94 & - & & & 2,09 & 8,3 & & & & & \\
\hline 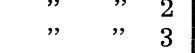 & $\begin{array}{l}82,14 \\
83,29\end{array}$ & $\begin{array}{l}15,84 \\
14,59\end{array}$ & 2,12 2,2 & - & & & $\begin{array}{l}2,07 \\
1,99\end{array}$ & $\begin{array}{l}8,4 \\
8,6\end{array}$ & & & & & \\
\hline $\begin{array}{lll}" & \# & 4 \\
" & & 5\end{array} \mid$ & $\begin{array}{l}80,10 \\
80,85\end{array}$ & $\begin{array}{l}19,26 \\
18,53\end{array}$ & $=$ & $\begin{array}{l}0,64 \\
0,62\end{array}$ & & & $\begin{array}{l}2,13 \\
2,07\end{array}$ & $\begin{array}{l}8,2 \\
8.4\end{array}$ & & & & & \\
\hline $\begin{array}{lll}\prime \prime & , & 0 \\
. & \end{array}$ & $\begin{array}{l}81,93 \\
8890\end{array}$ & $\begin{array}{l}17,48 \\
17,40\end{array}$ & - & 0,59 & & & $\begin{array}{l}2,12 \\
2,12\end{array}$ & $\begin{array}{l}8,4 \\
8,2\end{array}$ & & & & & \\
\hline $\begin{array}{lll}\prime & \cdots & 7 \\
" & 8\end{array} \mid$ & $\begin{array}{l}80,22 \\
80,97\end{array}$ & $\begin{array}{l}19,78 \\
19,03\end{array}$ & $\bar{z}$ & 二 & & & $\begin{array}{l}2,07 \\
2,06\end{array}$ & $\begin{array}{l}8,4 \\
8.4\end{array}$ & & & & & \\
\hline $\begin{array}{lll}n & n & 9 \\
\end{array}$ & 82,05 & 17,95 & - & - & & & $\begin{array}{l}2,100 \\
2,1\end{array}$ & 8,3 & & & & & \\
\hline Fábrica n.o 5 & $\begin{array}{c}\text { Caliza } \\
\text { silúrica }\end{array}$ & $\begin{array}{l}\text { Marga } \\
\text { silúrica }\end{array}$ & & & & & & & & & & & \\
\hline $\begin{array}{lll}\text { Crudo } & n & 0 \\
\# & 2\end{array}$ & & & & & & & 1,78 & 9,4 & - & $94,-$ & 3,5 & 2,0 & $\mathrm{a}$ \\
\hline $\begin{array}{lll}\| & , & 3 \\
, & 3\end{array}$ & & & & & & & $\begin{array}{l}1,76 \\
1,78\end{array}$ & , & 二 & $\begin{array}{r}110,8 \\
82,6\end{array}$ & $\begin{array}{l}\begin{array}{l}4,7 \\
3,0\end{array} \\
-\end{array}$ & $\begin{array}{l}1,3 \\
2,5\end{array}$ & $\begin{array}{l}\mathrm{a} \\
\mathrm{a}\end{array}$ \\
\hline 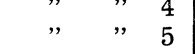 & & & & & & & $\begin{array}{l}1,84 \\
1,76\end{array}$ & $\begin{array}{l}9,2 \\
9,5\end{array}$ & 二 & $\begin{array}{l}84,5 \\
95,6\end{array}$ & $\begin{array}{l}3,1 \\
3,1\end{array}$ & $\begin{array}{l}2,1 \\
1.9\end{array}$ & $\begin{array}{l}\mathrm{a} \\
\mathrm{a}\end{array}$ \\
\hline $\begin{array}{lll}\prime, & , & 6 \\
\end{array}$ & & & & & & & $\begin{array}{l}1,75 \\
1,84\end{array}$ & $\begin{array}{l}9,6 \\
9,2\end{array}$ & $=$ & $\begin{array}{l}89,5 \\
, 0,5\end{array}$ & $\begin{array}{l}3,3 \\
28\end{array}$ & $\begin{array}{l}1,9 \\
1,5\end{array}$ & a \\
\hline $\left.\begin{array}{lll}\prime, & \# & 8 \\
, & 9\end{array}\right]$ & & & & & & & $\begin{array}{l}1,84 \\
1,63\end{array}$ & 9,2 & - & $\begin{array}{l}91,7 \\
91,7\end{array}$ & $\begin{array}{l}3,6 \\
3,6\end{array}$ & $\begin{array}{l}2,0 \\
1,6\end{array}$ & a \\
\hline$" \quad " 10$ & & & & & & & 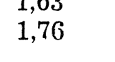 & $\begin{array}{r}r, 1,1 \\
9,5\end{array}$ & - & $\begin{array}{l}3,0 \\
94,9\end{array}$ & 年, & $\begin{array}{l}1,0 \\
1,9\end{array}$ & $\begin{array}{l}\mathrm{a} \\
\mathrm{a}\end{array}$ \\
\hline Fábrica no. 6 & $\begin{array}{l}\text { Caliza } \\
\text { mioceno }\end{array}$ & $\begin{array}{l}\text { Caolin } \\
\text { Albenia } \\
\text { cretácica }\end{array}$ & & & & & & & & & & & \\
\hline Crudo $n .{ }^{\circ} 1$ & & & & & & & 2,06 & 8,4 & & & & & \\
\hline Fábrica n.o 7 & $\begin{array}{c}\text { Caliza } \\
\text { Jurásica }\end{array}$ & $\begin{array}{l}\text { Arenisca } \\
\text { arcillosa }\end{array}$ & $\begin{array}{l}\text { Arenisca } \\
\text { arcillosas }\end{array}$ & Fscoria & & & & & & & & & \\
\hline & Dogger & mioceno & triásica & & & & & & & & & & \\
\hline $\begin{array}{lll}\text { Crudo } & n, 0 & 1 \\
, & 2\end{array}$ & $\begin{array}{l}80,12 \\
7907\end{array}$ & 19,88 & 83 & - & & & $\begin{array}{l}0,99 \\
099\end{array}$ & $\begin{array}{l}15,3 \\
153\end{array}$ & & & & & \\
\hline $\begin{array}{lll}\prime \prime & \quad & 3 \\
" & & 4\end{array}$ & 74,00 & 18,0 & 二 & 8,0 & & & $\begin{array}{l}1,00 \\
1,03\end{array}$ & $\begin{array}{l}15,2 \\
14,3\end{array}$ & & & & & \\
\hline
\end{tabular}




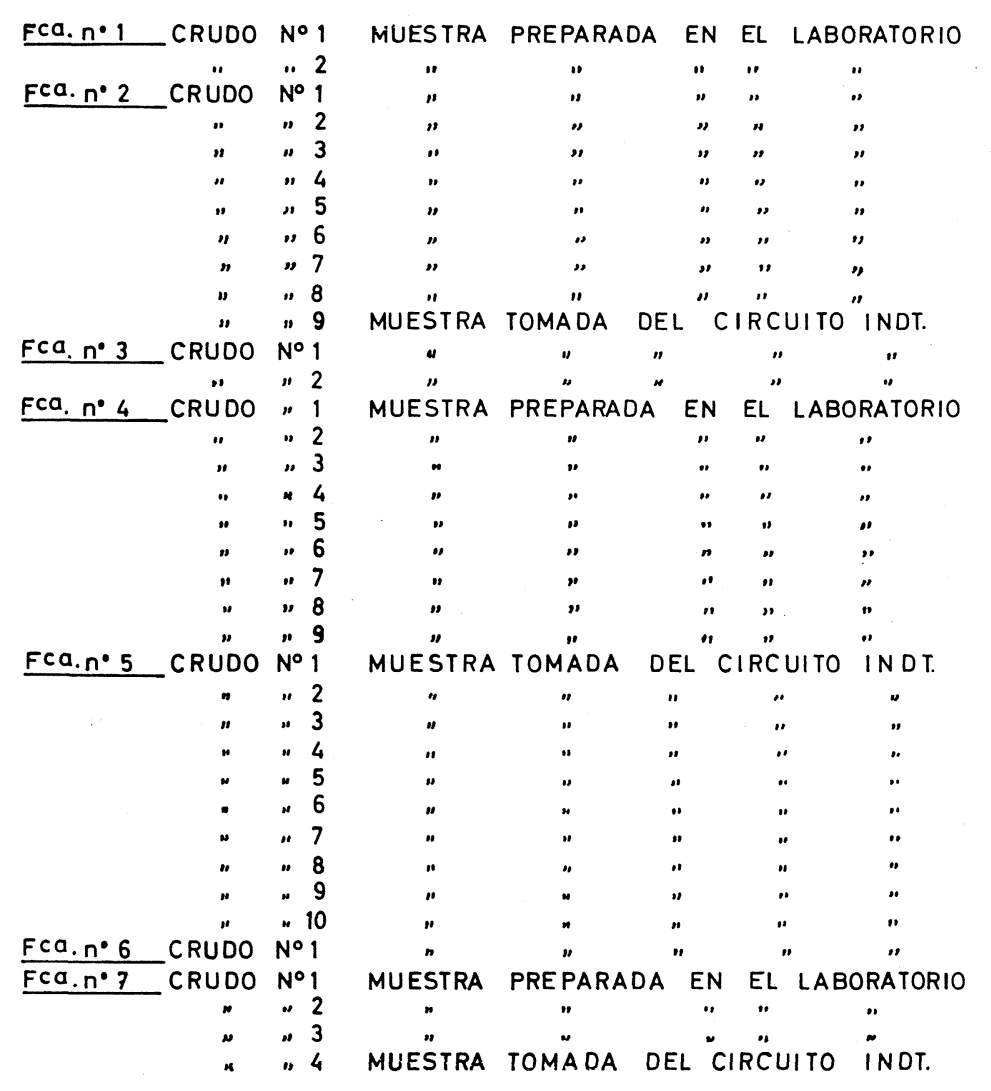

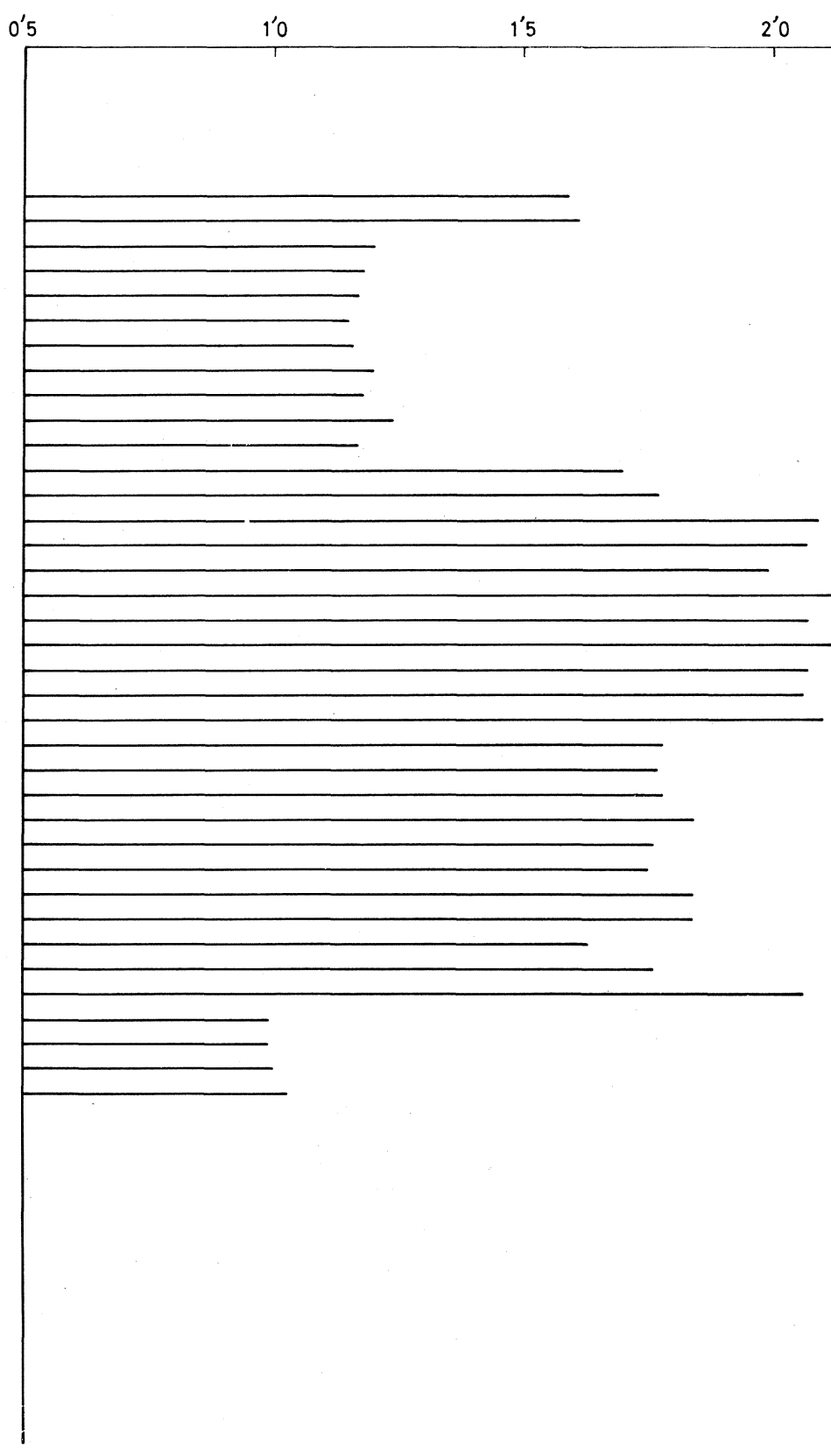

GRAFICO II a 


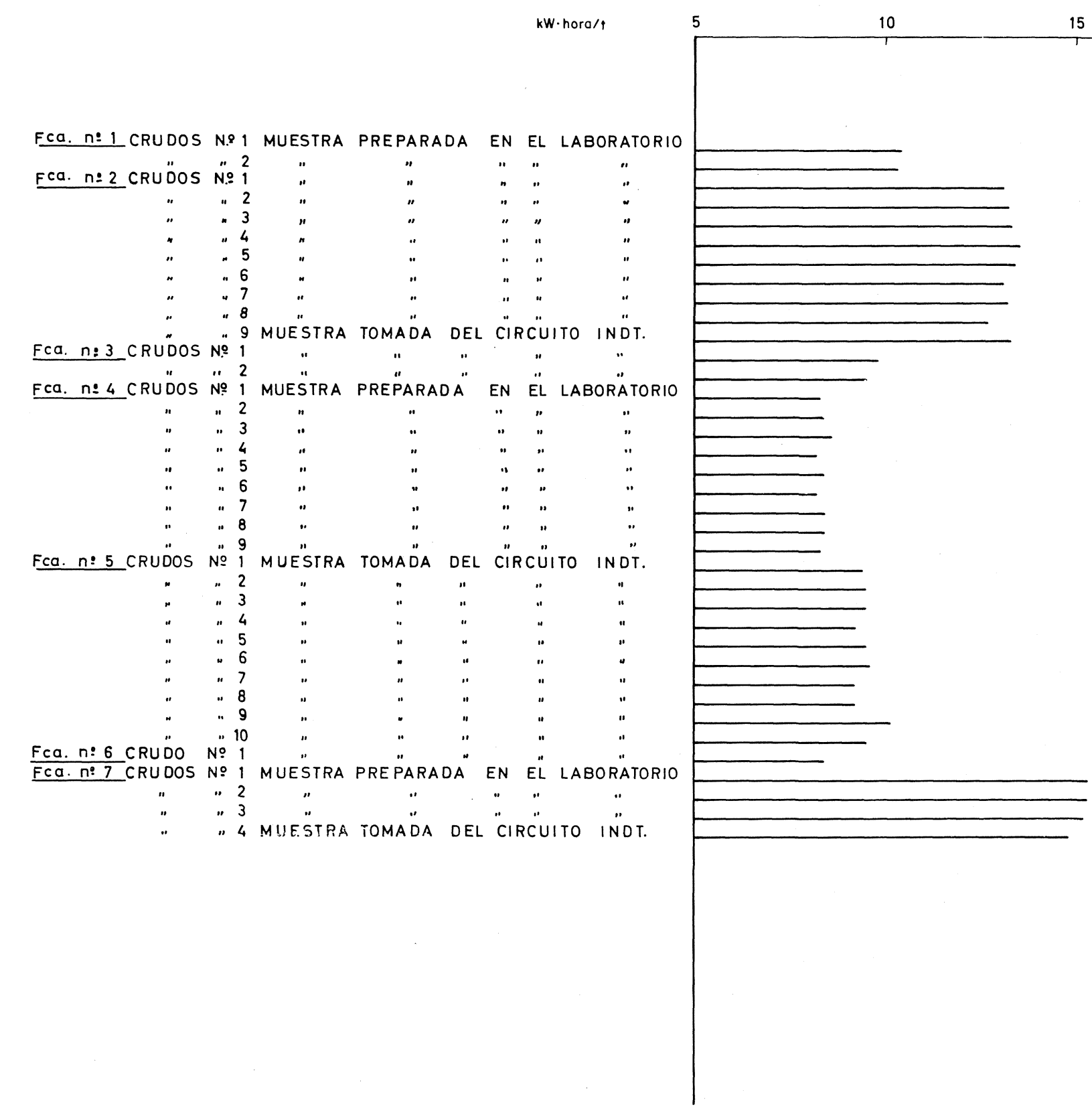

GRAFICO II b 
FCa. N:2 CLINKER H. No. 2 MUESTRA No1

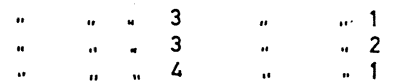

$$
\begin{aligned}
& 95 \% " \text { CLINKER" H. N N } 2 \text { " }+5 \% \text { "YESO } \\
& 95 \% " \quad-3+5 \% "
\end{aligned}
$$

FE. N. 3 CLINKER "PROME"DIO" HORNOS

$90 \%$ "CLINKER CON $5 \%$ YESO MAS $10.0 \%$ PUZOLANA ARTIFICIAI FEa. N. 4 CLINKER "H. NO $1 "$

$$
" \quad " \quad " \quad 2 \quad 1+5 \% \text { YESO }
$$

$72 \%$ " CLINKERR H. NO 1 CON $5 \%$ YESO + $28 \%$ LIMBURGITAS (C.REAL:

$72 \%$ " " 2 " $5 \%$ " $5 \%$

$68 \% \quad 5 \%$

$72 \% \quad " \quad 1,5 \%,+28 \%$ PUZOL̈ANA ARTIFICIAI

$72 \%$

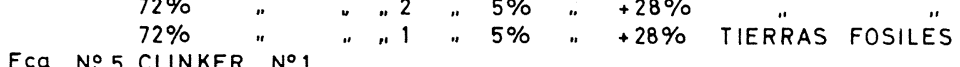

Fca. N. 5 CLINKER No

$$
\begin{aligned}
& \text { : } \quad 33 \\
& \begin{array}{r}
2 \\
3 \\
4 \\
5 \\
6 \\
7 \\
8 \\
9 \\
10
\end{array} \\
& \begin{array}{l}
.79 \\
.10 \\
.1
\end{array} \\
& \text { " H. N० } 1 \text { MUESTRA } \text { No1 }^{\circ}
\end{aligned}
$$

Fca. N. 6

FEa. N:7 " STOCK MUESTRA N01

$g /$ rev.

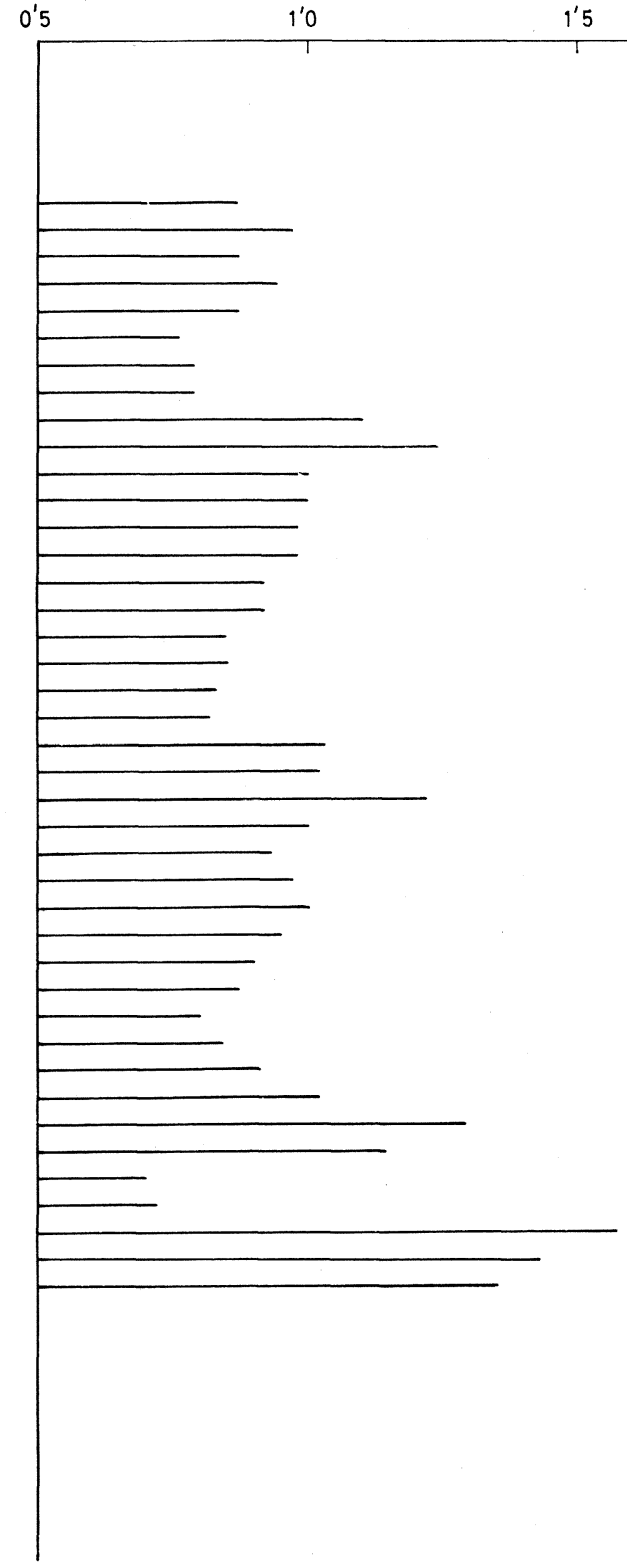


TAвLA III

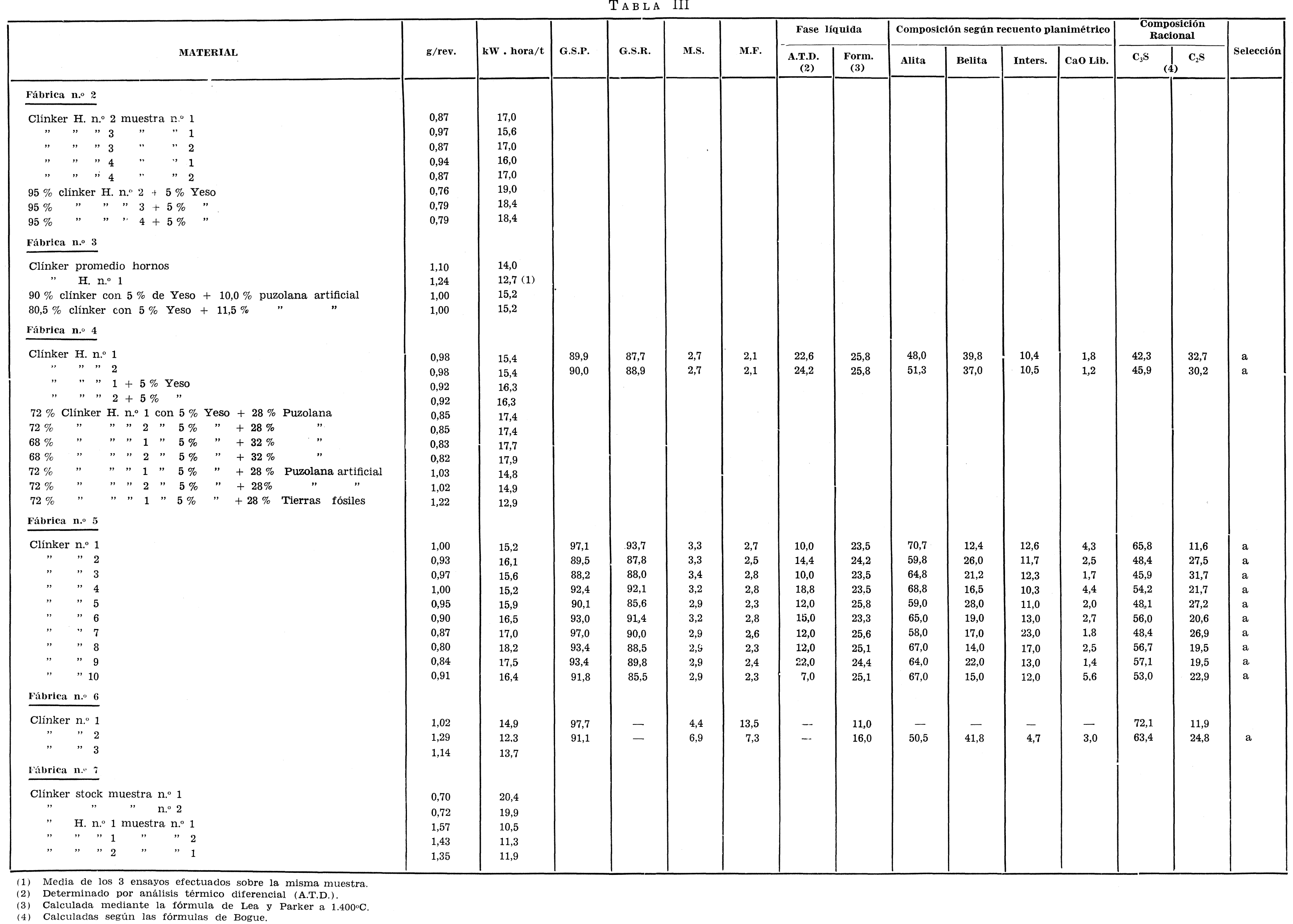


FE. N.2 CLINKER H. № 2 MUESTRA NN 1

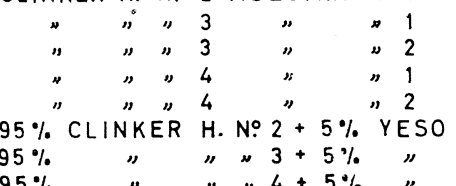

" $3+5 \%$ " 3 "

$95 \%$
CLINKER PROMEDIO HORNOS

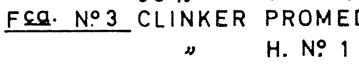

H. NO 1
$90 \%$ CLINKER CON $5 \%$ YESO + $10 \%$ DE PUZOLANA ARTIFICIAL Fç. N. 4 CLINKER "H. N. 1 " 1

" " " $1+5 \%$ YESO

$2 \%$ " CLINKER "H. NO 1 1 CON $5 \%$ YESO + $28 \%$ LIMBURGITAS (C. REAL)

$72 \%$ " " 2 " $2 \% " 5 \%$

$68 \%$ " " 1 " $5 \%$ " $+32 \%$

$72 \%$ " " " 72 "

" " " " $2 \%$ "

$72 \% "$ " $72 \%$ "

FEQ. N. 5 CLINKER" N. 1

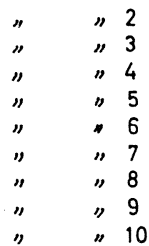

F c. N. 06 CLINKER "N: 1

$$
\text { " " } 2
$$

F@Q. NO 7 CLINKER STOCK MUESTRA NN 1

$$
\begin{aligned}
& \text { " H. NO } 1 \text { MUESTRA "No } 1 \\
& \text { " " ग }
\end{aligned}
$$

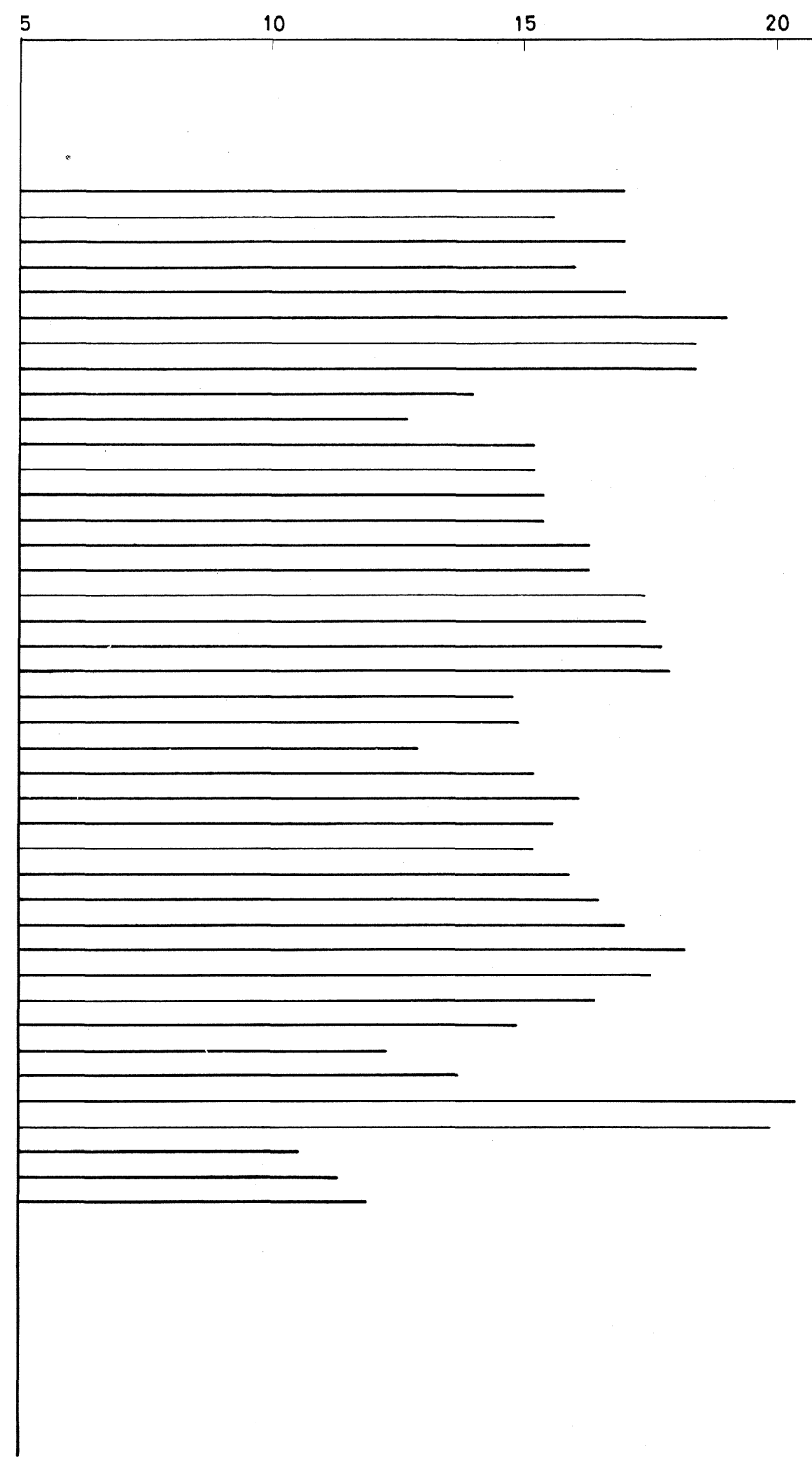

GRAFICO III b 
TABLA III bis

\begin{tabular}{|c|c|c|c|c|c|c|c|c|c|c|c|c|c|}
\hline & & \multirow{2}{*}{ G.S.P. } & \multirow{2}{*}{ G.S.R. } & \multirow{2}{*}{ M.S. } & \multirow{2}{*}{ MF. } & \multicolumn{2}{|c|}{ Fase líquida } & \multirow{2}{*}{ Alita } & \multicolumn{2}{|c|}{ Recuento planimétrico } & \multirow{2}{*}{ CaO libre } & \multicolumn{2}{|c|}{$\begin{array}{c}\text { Composición Racional } \\
\text { (CR) }\end{array}$} \\
\hline & & & & & & ATD & Form. & & Belita & Inters. & & $\mathrm{C}_{3} \mathrm{~S}$ & $\mathrm{C}_{2} \mathrm{~S}$ \\
\hline Total & $\begin{array}{l}\text { Coef. corr. } \\
\text { Pendiente } \\
\text { Ord. orig. } \\
\text { T. cal. } \\
\text { T. tabl. }\end{array}$ & $\begin{array}{r}0,15 \\
0,07 \\
93,72 \\
\text { no }\end{array}$ & $\begin{array}{c}-0,16 \\
-0,06 \\
21,80 \\
\text { no }\end{array}$ & $\begin{array}{c}-0,78 \\
-1,0 \\
19,22 \\
\mathrm{si}\end{array}$ & $\begin{array}{c}-0,48 \\
-0,22 \\
16,61 \\
\text { no }\end{array}$ & $\begin{array}{c}-0,15 \\
-0,03 \\
16,60 \\
\text { no }\end{array}$ & $\begin{array}{r}0,58 \\
0,19 \\
011,43 \\
\text { si }\end{array}$ & $\begin{array}{r}0,44 \\
0,09 \\
10,65 \\
\text { no }\end{array}$ & $\begin{array}{c}-0,61 \\
-0,09 \\
17,97 \\
\text { si }\end{array}$ & $\begin{array}{r}0,76 \\
0,26 \\
12,66 \\
\mathrm{si}\end{array}$ & $\begin{array}{r}-0,17 \\
-0,19 \\
16,41 \\
\text { no }\end{array}$ & $\begin{array}{r}-0,28 \\
-0,05 \\
18,31 \\
\text { no }\end{array}$ & $\begin{array}{c}-0,06 \\
-0,01 \\
16,12 \\
\text { no }\end{array}$ \\
\hline Selección (a) & $\begin{array}{l}\text { Coef. corr. } \\
\text { Pendiente } \\
\text { Ord. orig. } \\
\text { T. cal. } \\
\text { T. tabl. }\end{array}$ & $\begin{array}{r}0,29 \\
0,15 \\
1,98 \\
\text { no }\end{array}$ & & $\begin{array}{c}-0,78 \\
-1,0 \\
19,24 \\
\mathrm{si}\end{array}$ & $\begin{array}{c}-0,76 \\
-0,80 \\
18,17 \\
\mathrm{si}\end{array}$ & & $\begin{array}{c}0,75 \\
0,41 \\
6,01 \\
\mathrm{si}\end{array}$ & $\begin{array}{r}0,45 \\
0,09 \\
10,65 \\
\text { no }\end{array}$ & $\begin{array}{c}-0,61 \\
-0,09 \\
17,97 \\
\mathrm{si}\end{array}$ & $\begin{array}{r}0,76 \\
0,26 \\
12,66 \\
\mathrm{si}\end{array}$ & $\begin{array}{c}-0,18 \\
-0,19 \\
16,41 \\
\text { no }\end{array}$ & $\begin{array}{r}-0,21 \\
-0,04 \\
18,09 \\
\text { no }\end{array}$ & $\begin{array}{c}-0,19 \\
-0,04 \\
16,99 \\
\text { no }\end{array}$ \\
\hline Selección (a) & $\begin{array}{l}\mathrm{kW} \text {. hora/t } \\
\text { Coef. corr. } \\
\text { F. cal. } \\
\text { F. tabl. } \\
\text { F, to5/0,01) }\end{array}$ & & & $\begin{array}{c}\text { G.S.P. } \\
\text { M.S.S. } \\
0,81 \\
\text { si } / \text { si }\end{array}$ & $\begin{array}{c}\text { G.S.P. } \\
\text { M.F. } \\
0,81 \\
\text { si } / \mathrm{si}\end{array}$ & & $\begin{array}{l}\text { G.S.P. } \\
\text { Fi Form. } \\
0,79 \\
\text { si } / \text { si }\end{array}$ & $\begin{array}{c}\text { G.S.P. } \\
\text { CR Alita } \\
0,46 \\
\text { no/no }\end{array}$ & $\begin{array}{c}\text { G.S.P. } \\
\text { CR Belita } \\
0,62 \\
\text { no/no }\end{array}$ & $\begin{array}{c}\text { G.S.P. } \\
\text { CR Inters. } \\
0,79 \\
\text { si } / \mathrm{si}\end{array}$ & $\begin{array}{l}\text { G.S.P. } \\
\mathrm{CaO} i . \\
0,39 \\
\text { no/no }\end{array}$ & $\begin{array}{r}\text { G.S.P. } \\
\text { F.C. } \\
0,55 \\
\text { no/no }\end{array}$ & 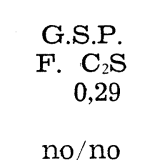 \\
\hline Selección !(a) & $\begin{array}{l}\mathrm{kW} \text {. hora/t } \\
\text { Coef. corr. } \\
\text { F. cal. } \\
\text { F. tabl. } \\
\text { (0,05/0,01) }\end{array}$ & & & & $\begin{array}{l}\text { M.S. } \\
\text { M.F. } \\
0,78 \\
\text { si } / \mathrm{si}\end{array}$ & & $\begin{array}{l}\text { M.S. } \\
\text { Fil Form. } \\
0,78 \\
\text { Si } / \text { si }\end{array}$ & $\begin{array}{c}\text { M.S. } \\
\text { CR Alita } \\
0,81 \\
\text { si } / \text { si }\end{array}$ & $\begin{array}{c}\text { M.S. } \\
\text { CR Belita } \\
0,84 \\
\text { si } / \text { si }\end{array}$ & $\begin{array}{l}\text { M.S. } \\
\text { CR Inters. } \\
0,87 \\
\text { si } / \text { si }\end{array}$ & $\begin{array}{c}\text { M.S. } \\
\mathrm{CaO} i . \\
0,78 \\
\text { si/si }\end{array}$ & $\begin{array}{l}\text { M.S. } \\
\text { F. C. } \\
0,81 \\
\text { si } / \text { si }\end{array}$ & $\begin{array}{l}\text { M.S. } \\
\text { F. . . }{ }_{2} \text { S } \\
0,81 \\
\text { si /si }\end{array}$ \\
\hline Selección (a) & $\begin{array}{l}\mathrm{kW} \text {. hora/t } \\
\text { Coef. corr. } \\
\text { F. cal. } \\
\text { F. tabl. } \\
\text { (0,05/0,01) }\end{array}$ & & & & & & $\begin{array}{l}\text { M.F. } \\
\text { Fl Form. } \\
0,76 \\
\text { si } / \text { no }\end{array}$ & $\begin{array}{c}\text { M.F. } \\
\text { CR Alita } \\
0,80 \\
\text { si/si }\end{array}$ & $\begin{array}{c}\text { M.F. } \\
\text { CR Belita } \\
0,83 \\
\text { Si } / \text { si }\end{array}$ & $\begin{array}{l}\text { M.F. } \\
\text { CR Inters. } \\
0,88 \\
\text { si } / \text { si }\end{array}$ & $\begin{array}{c}\text { M.F.F. } \\
\mathrm{CaO} i \\
0,77 \\
\text { si } / \mathrm{no}\end{array}$ & $\begin{array}{l}\text { M.F. } \\
\text { F. C.S } \\
0,79 \\
\text { si } / \text { si }\end{array}$ & $\begin{array}{l}\text { M.F. } \\
\text { F. } C_{2} \text {.S } \\
0,80 \\
\text { si } / \text { si }\end{array}$ \\
\hline Selección (a) & $\begin{array}{l}\mathrm{kW} \text {. hora/t } \\
\text { Coof. corr. } \\
\text { F. cal. } \\
\text { F. tabl. } \\
(0,05 / 0,01)\end{array}$ & & & & & & & $\begin{array}{c}\text { Fl Form. } \\
\text { CR Alita } \\
0,82 \\
\text { si } / \mathrm{si}\end{array}$ & $\begin{array}{c}\text { Fl Form. } \\
\text { CR Belita } \\
0,85 \\
\text { si } / \mathrm{si}\end{array}$ & $\begin{array}{c}\text { Fi Form. } \\
\text { CR Inters. } \\
0,85 \\
\text { Si } / \text { si }\end{array}$ & $\begin{array}{c}\text { Fi Form. } \\
\text { CaO } l \text {. } \\
0,75 \\
\text { si/no }\end{array}$ & $\begin{array}{c}F 1 \text { Form. } \\
\text { F. } C_{\mathrm{S} S} \\
0,81 \\
\mathrm{si} / \mathrm{si}\end{array}$ & $\begin{array}{c}\mathrm{Fl} \text { Form. } \\
\mathrm{F} . \mathrm{C}_{2, \mathrm{~S}} \\
0,81 \\
\mathrm{si} / \mathrm{si}\end{array}$ \\
\hline Selección , (a) & $\begin{array}{l}\mathrm{kW} \text {. hora/t } \\
\text { Coef. corr. } \\
\text { F. cal. } \\
\text { F. tabl. } \\
(0,05 / 0,01)\end{array}$ & & & & & & & & $\begin{array}{c}\text { CR Alita } \\
\text { CR Belita } \\
0,70 \\
\text { si } / \text { no }\end{array}$ & $\begin{array}{c}\text { CR Alita } \\
\text { CR Inters. } \\
0,80 \\
\text { si } / \mathrm{si}\end{array}$ & $\begin{array}{c}\text { CR Alita } \\
\text { CaO l. } \\
0,68 \\
\text { si } / \text { no }\end{array}$ & $\begin{array}{c}\underset{\mathrm{CR} . \mathrm{Alita}}{\mathrm{F} \cdot \mathrm{C}, \mathrm{S}} \\
0,64 \\
\mathrm{no} / \mathrm{no}\end{array}$ & $\begin{array}{c}\text { CR. Alita } \\
\text { F. } \mathrm{C}_{25} \text {, } \\
0,48 \\
\text { no/no }\end{array}$ \\
\hline Selección '(a) & $\begin{array}{l}\mathrm{kW} \text {. hora/t } \\
\text { Coef. corr. } \\
\mathrm{F} . \text { cal. } \\
\text { F. tabl. } \\
(0,05 / 0,01)\end{array}$ & & & & & & & & & $\begin{array}{c}\text { CR Belita } \\
\text { CR Inters. } \\
0,79 \\
\text { si } / \text { si }\end{array}$ & $\begin{array}{c}\text { CR Belita } \\
\text { CaO l. } \\
0,81 \\
\text { si } / s i\end{array}$ & $\begin{array}{c}\text { CR Belita } \\
\text { F. C.S } \\
0,75 \\
\text { si } / \text { no }\end{array}$ & $\begin{array}{c}\text { CR. Belita } \\
\text { F. C } \mathrm{C}_{2} \mathrm{~S} \\
0,67 \\
\text { no/no }\end{array}$ \\
\hline Selección (a) & 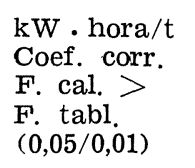 & & & & & & & & & & $\begin{array}{c}\text { CR Inters. } \\
\text { CaO l. } \\
0,76 \\
\text { si } / \text { no }\end{array}$ & $\begin{array}{c}\text { CR Inters. } \\
\text { F. C. } \mathrm{C}_{3} \mathrm{~S} \\
0.77 \\
\text { si } / \text { no }\end{array}$ & $\begin{array}{c}\text { CR Inters. } \\
\text { F. } \mathrm{C}_{25} \text {. } \\
0,77 \\
\text { si } / \text { no }\end{array}$ \\
\hline Selección (a) & $\begin{array}{l}\mathrm{kW} \text {. hora/t } \\
\text { Coef. corr. } \\
\text { F. cal. } \\
\text { F. tabl. } \\
\text { (0,05/0,01) }\end{array}$ & & & & & & & & & & & $\begin{array}{c}\mathrm{CaO} l \\
\mathrm{Ca} \cdot \mathrm{C}, \mathrm{S} \\
0,22 \\
\mathrm{no} / \mathrm{no}\end{array}$ & $\begin{array}{l}\mathrm{CaO} l . \\
\mathrm{F} \cdot \mathrm{C}_{2} \mathrm{~S} \\
0,38 \\
\text { no/no }\end{array}$ \\
\hline Selección (a) & $\begin{array}{l}\mathrm{kW} \text {. hora/t } \\
\text { Coef. corr. } \\
\text { F. cal . }> \\
\text { F. tabl. } \\
(0,05 / 0,01)\end{array}$ & & & & & & & & & & & & $\begin{array}{c}\mathrm{F} \cdot \mathrm{C}_{\mathrm{S} S} \\
\mathrm{~F} \cdot \mathrm{C}_{2 \mathrm{~S}} \\
0,79 \\
\mathrm{si} / \mathrm{si}\end{array}$ \\
\hline
\end{tabular}



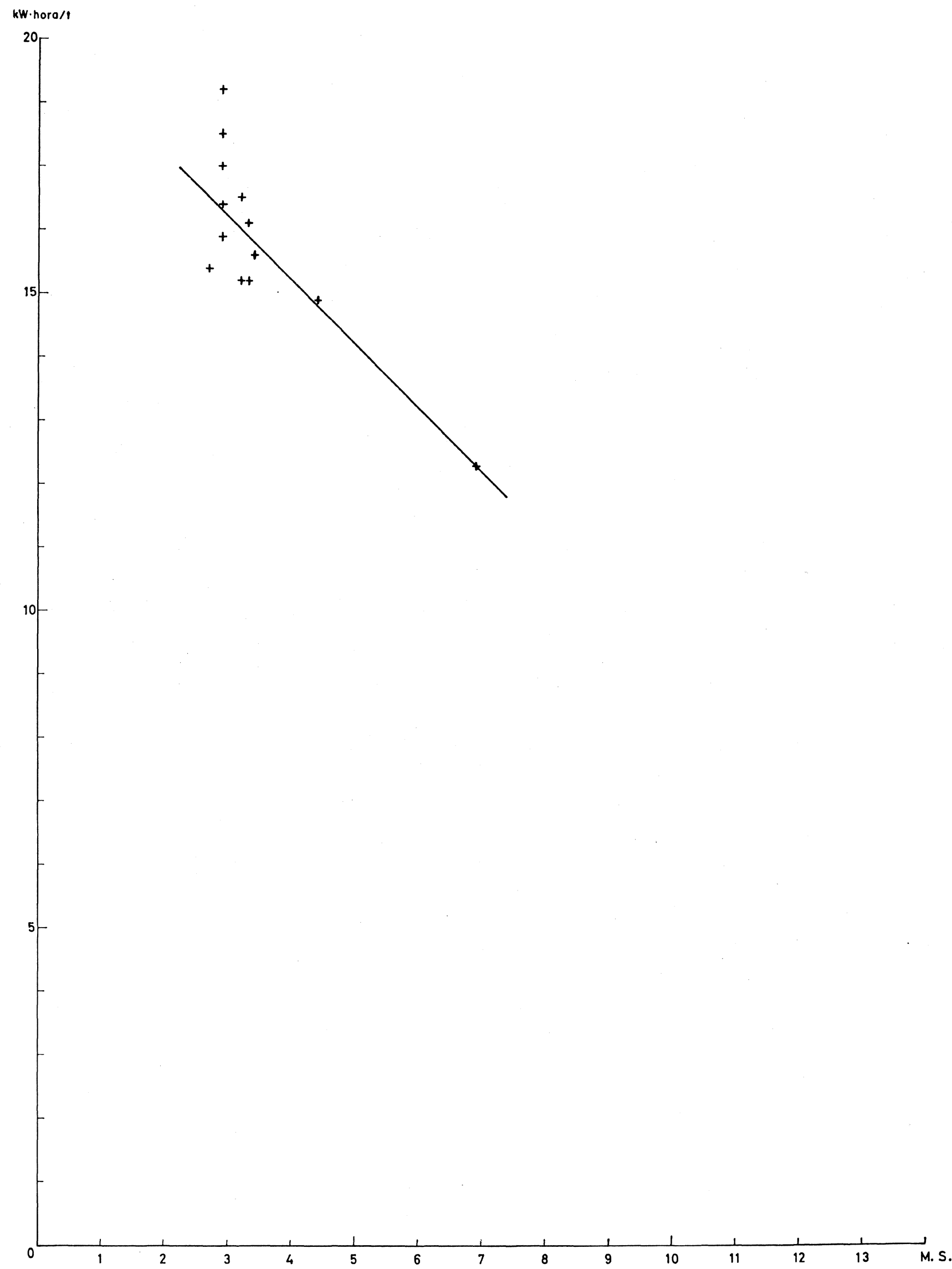

GRAFICO III c 


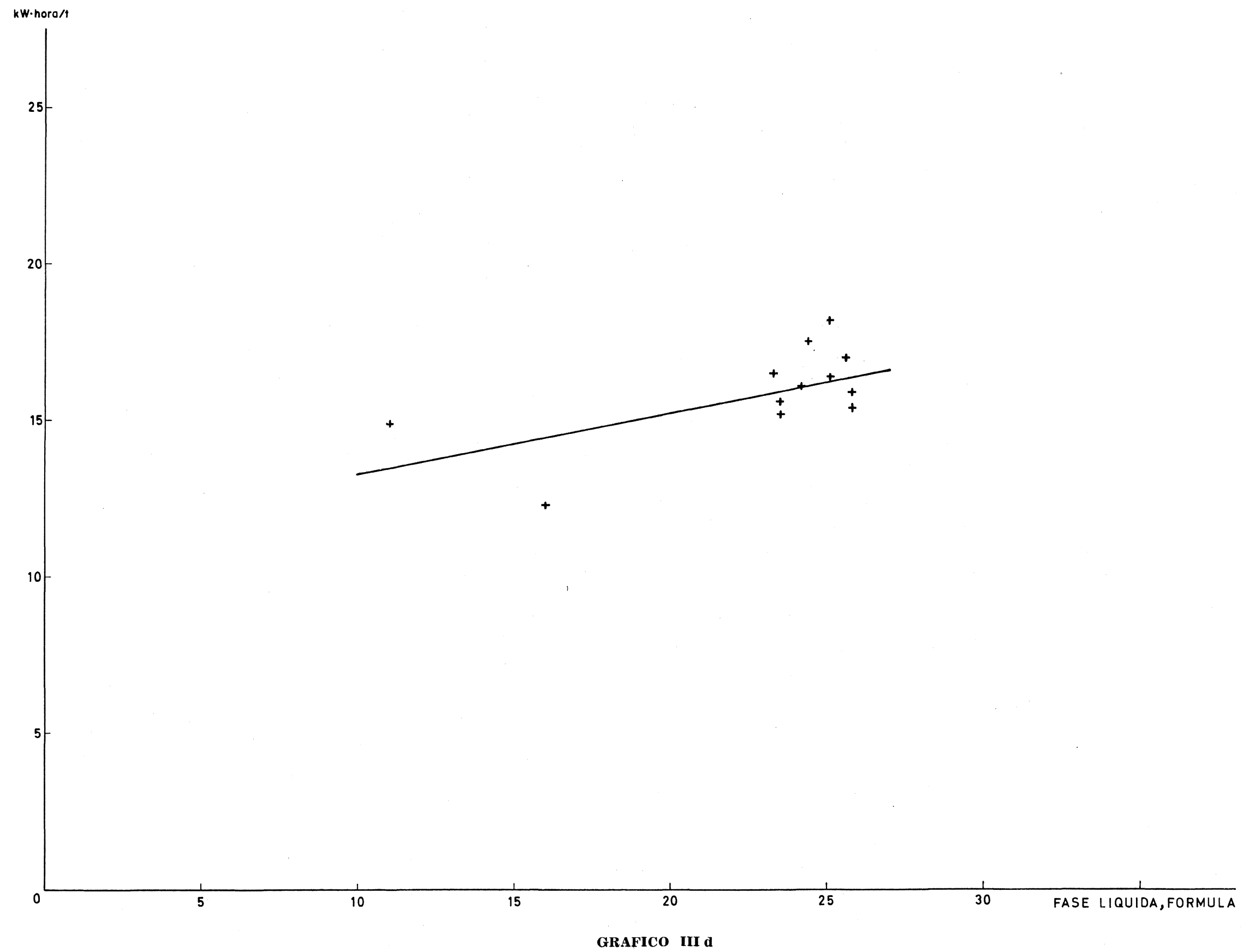

(c) Consejo Superior de Investigaciones Científicas Licencia Creative Commons 3.0 España (by-nc) 


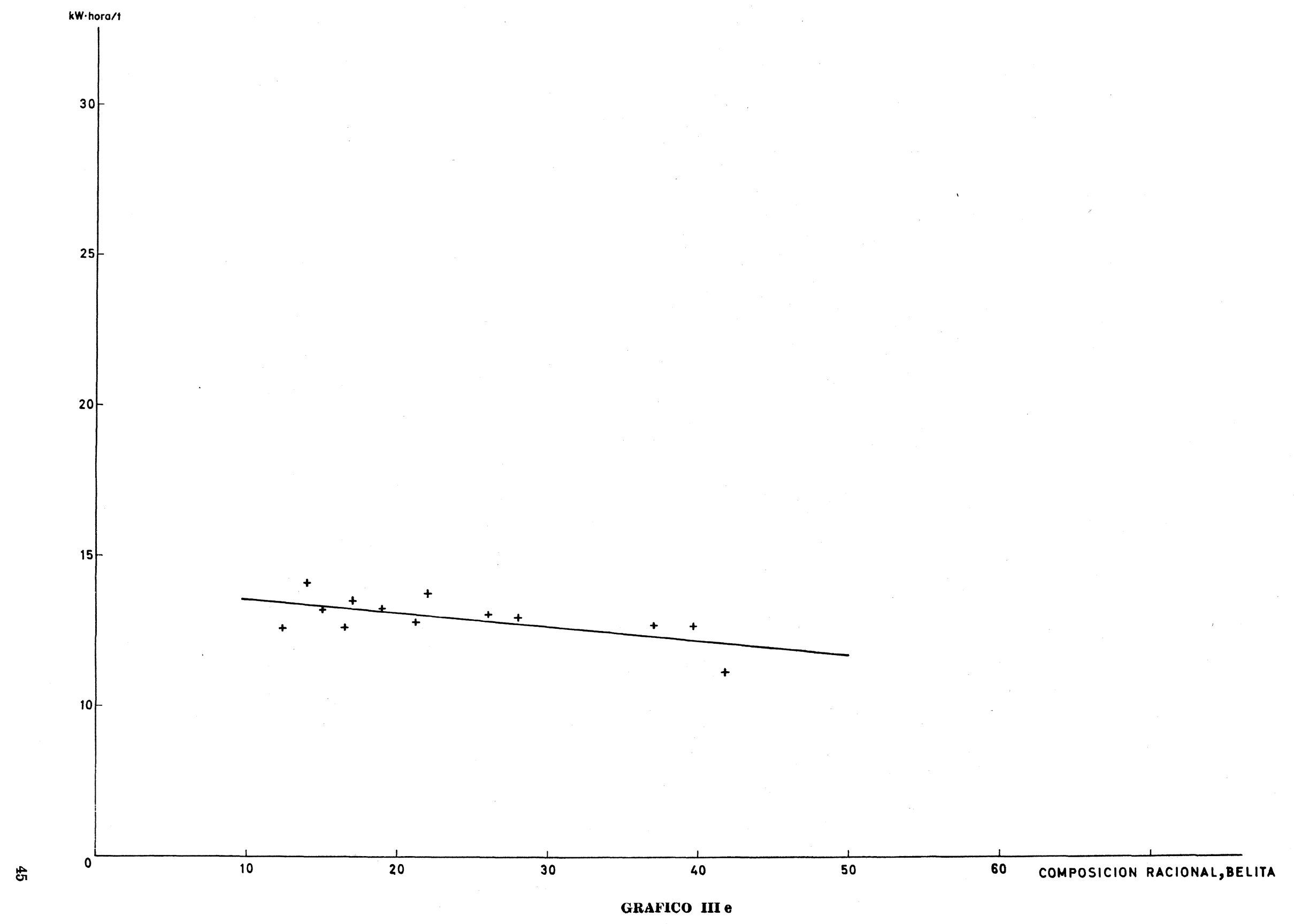

(c) Consejo Superior de Investigaciones Científicas Licencia Creative Commons 3.0 España (by-nc) 


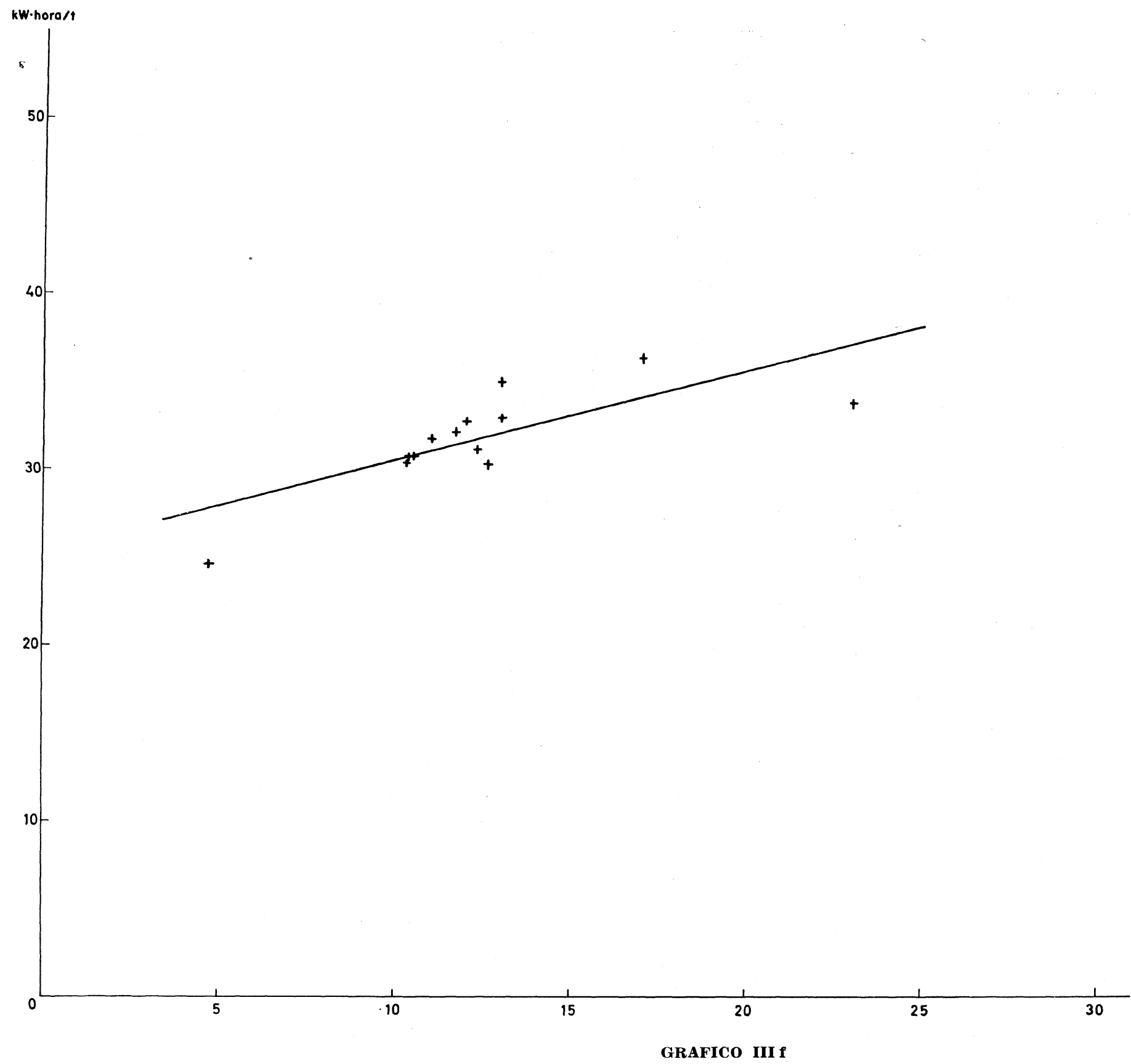

COMPOSICION RACIONAL,

FASE INTERSTICIAL

(c) Consejo Superior de Investigaciones Científicas Licencia Creative Commons 3.0 España (by-nc)

http://materconstrucc.revistas.csic.es 
T A B L A IV

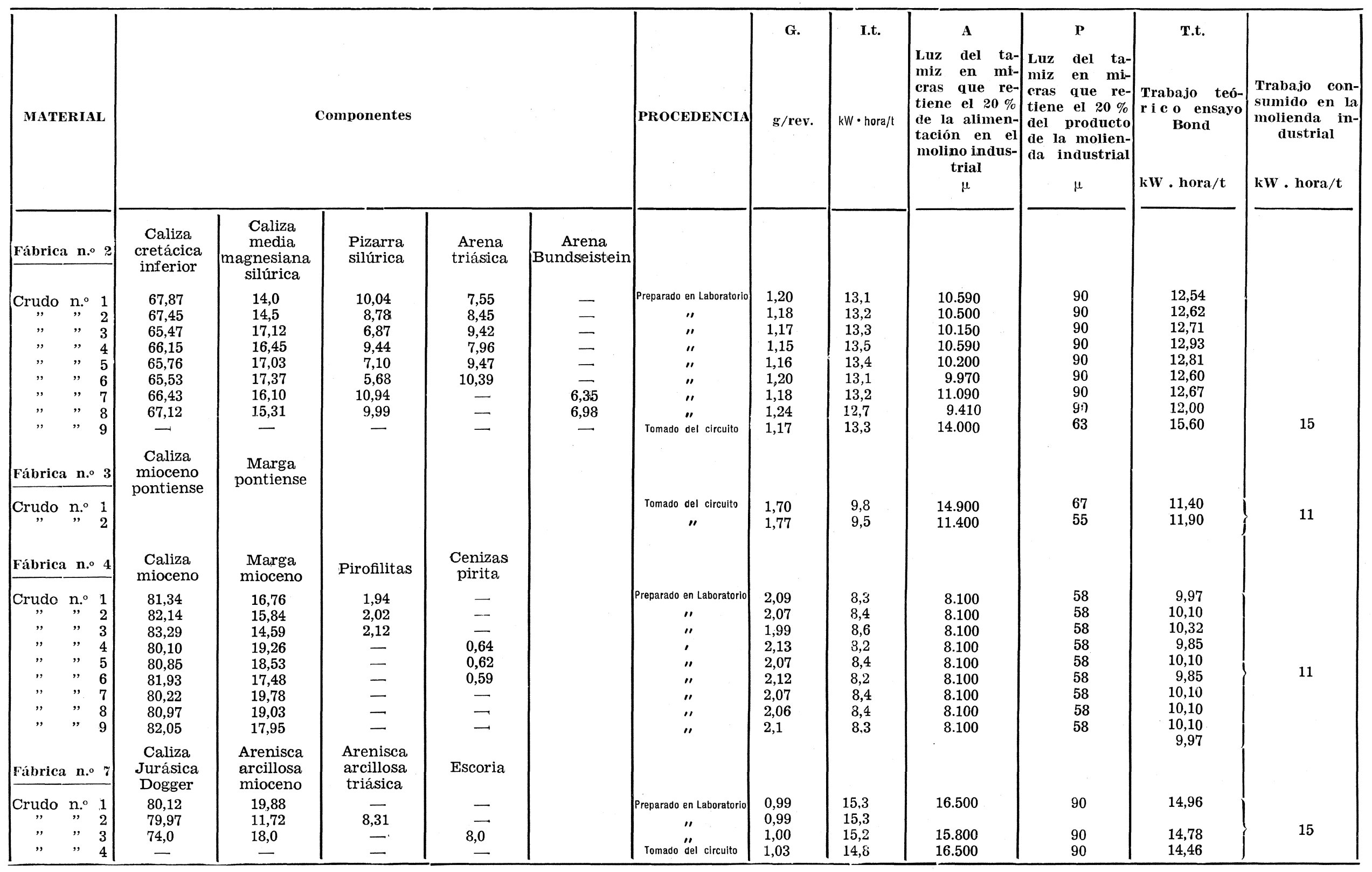


Tabla $\mathrm{V}$

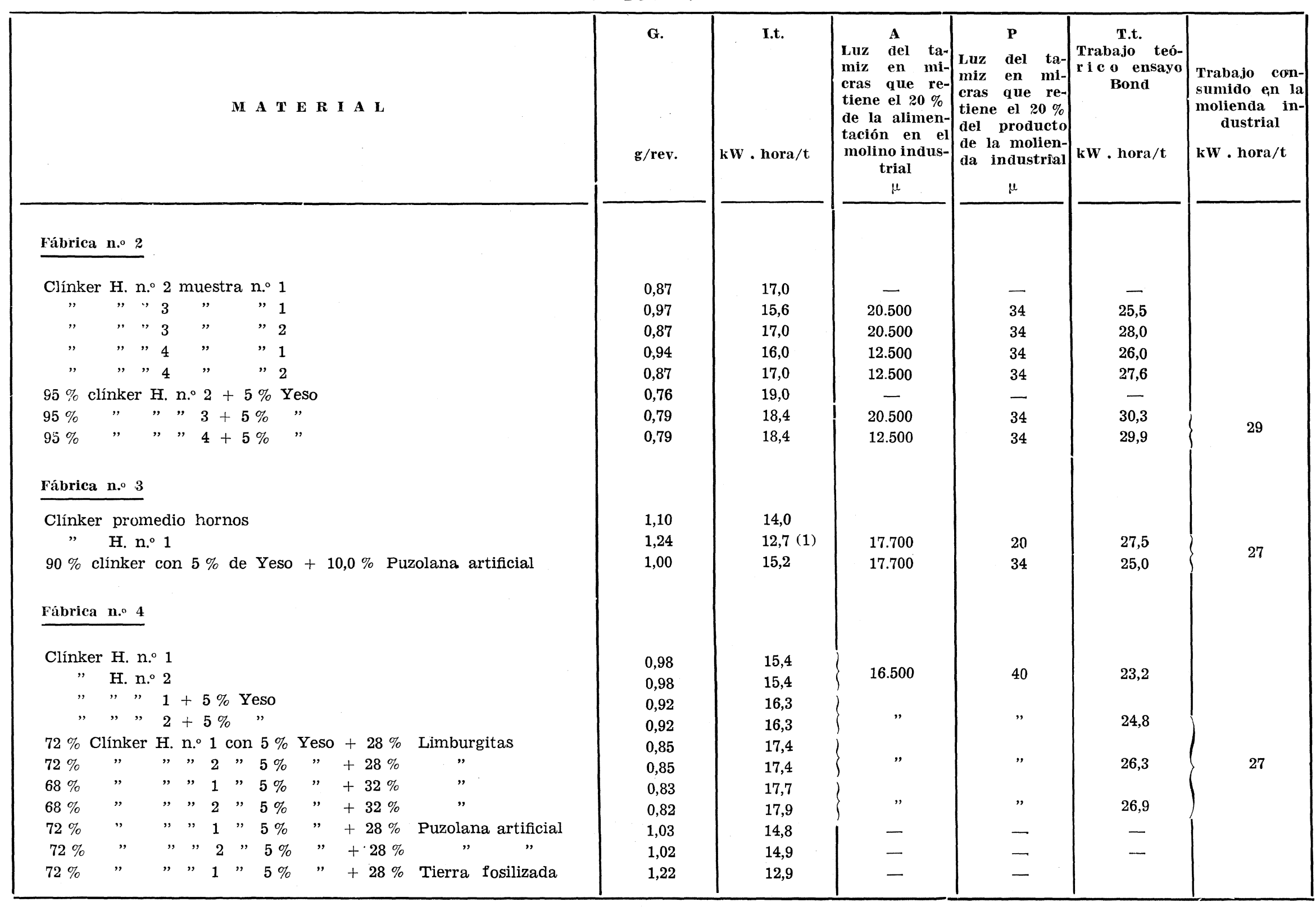



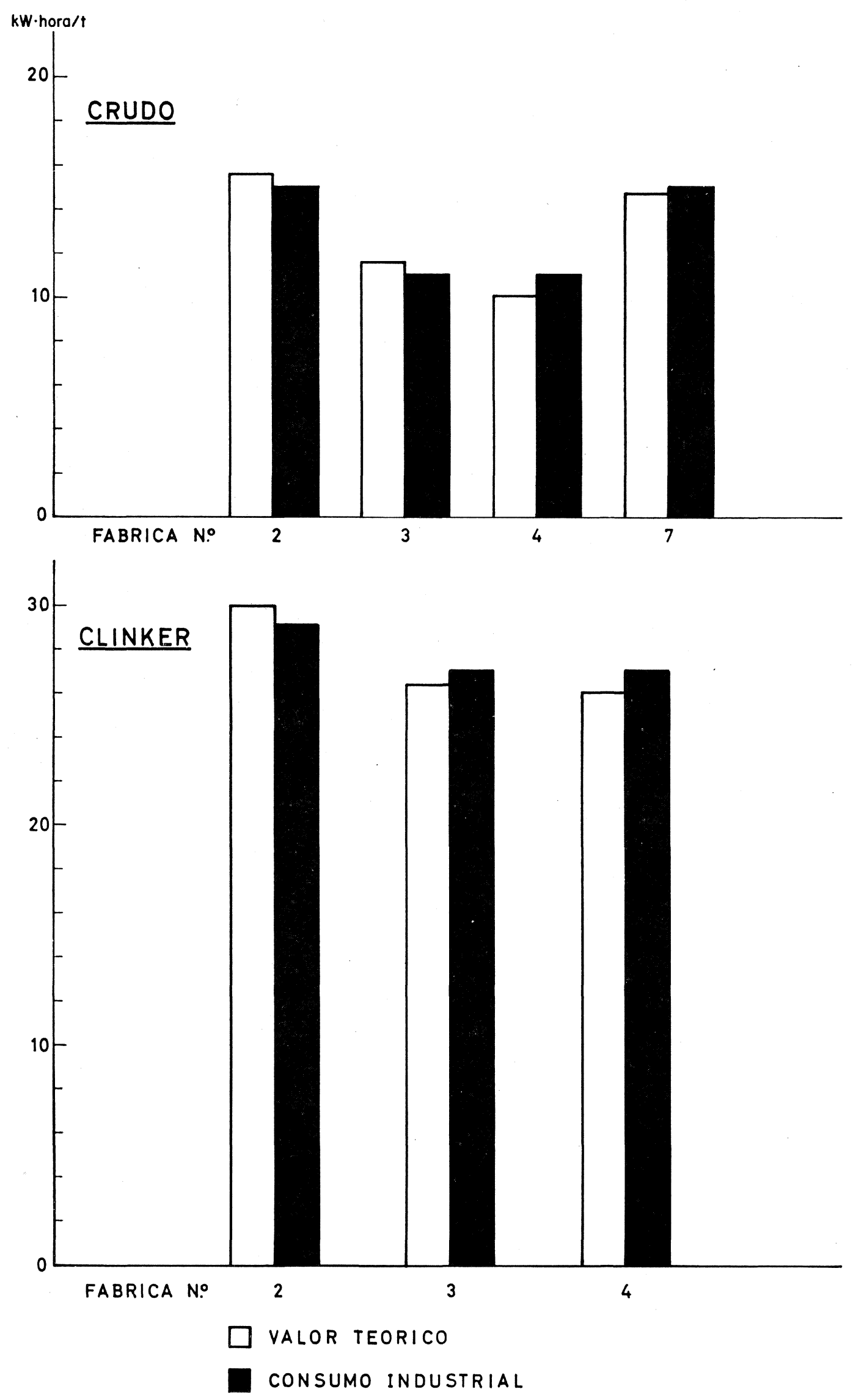

GRAFICO VII 
Deduciéndose que de las variables consideradas, las correlaciones más fuertes, son $\mathrm{kW}$. - hora/t con la fase intersticial y el módulo de fundentes, $\mathrm{kW} \cdot$ hora/t con la fase intersticial y el módulo de silicatos, y $\mathrm{kW}$. hora/t con la fase intersticial y el contenido de fase líquida según Lea y Parker.

\section{CONCORDANCIA DE RESULTADOS OBTENIDOS EN EL LABORATORIO CON LOS INDUSTRIALES}

Según lo expuesto en el método de ensayo (apartado 2.1.) hemos podido calcular, a partir del índice de trabajo determinado para $\gamma=90$, la potencia o trabajo teórico que un molino industrial requerirá para moler un determinado material, desde una determinada granulometría a otra.

No obstante debemos señalar que, tal como se establece en el tercer principio de la molienda, el índice de trabajo no es realmente una constante para cada material, sino que es función de la finura final de la molienda (o sea del tamiz separador y utilizado en el ensayo).

Por lo que el índice de trabajo empleado para calcular el trabajo teórico debería ser, si se quiere obtener un valor exacto, el obtenido empleando en el ensayo "Bond Mill" el tamiz "separador $\gamma$ " para el cual se obtenga una granulometría del producto molido semejante a la planeada en la molienda industrial.

Los valores de los trabajos teóricos calculados para crudos y clínker, conjuntamente con los consumidos en la molienda industrial, vienen expuestos en las tablas IV y V respectivamente y representados gráficamente en forma de diagrama de barras en el gráfico VII.

De las que se desprende que, la concordancia entre los valores teóricos calculados según el método de F. C. Bond y los consumidos industrialmente, atendiendo únicamente al motor del molino, es francamente buena.

En consecuencia podemos decir que actualmente disponemos de un método aceptable para predecir el consumo energético en la molienda de los materiales de la industria del cemento, sin necesidad de efectuar el ensayo a escala industrial.

\section{BIBLIOGRAFIA CONSULTADA}

[1] As. Kannewurf.: Rocks Products, May (1957) pág. 86.

[2] HD Wasmuth.: Aufbereitungs-Technik, n.o 6, junio (1969), pág. 284.

[3] F. C. Bond.: British Chemical Engineering. June (1961) Vol. 6, n. 6, pág. 378 y n.º 8, pág. 543.

[4] Grampiefro Tognon.: Revista Ingeniería, n. 2, febrero 1957.

[5] Warreh Spruig Laboratory.: Mineral Processing Information. Note n. 3.

[6] F. C. Bond.: Cement Lime and Gravel, febrero 1971. 